\title{
Anomalies as obstructions: from dimensional lifts to swampland
}

\author{
Peng Cheng, ${ }^{a}$ Ruben Minasian ${ }^{a}$ and Stefan Theisen ${ }^{b}$ \\ ${ }^{a}$ Institut de Physique Théorique, Université Paris Saclay, \\ CNRS, CEA, F-91191, Gif-sur-Yvette, France \\ ${ }^{b}$ Max-Plank-Institut für Gravitationsphysik, \\ Albert-Einstein-Institut Am Mühlenberg 1, D-14476 Potsdam, Germany \\ E-mail: peng.cheng@ipht.fr, ruben.minasian@ipht.fr, \\ stefan.theisen@aei.mpg.de
}

ABSTRACT: We revisit the relation between the anomalies in four and six dimensions and the Chern-Simons couplings one dimension below. While the dimensional reduction of chiral theories is well-understood, the question which three and five-dimensional theories can come from a general circle reduction, and are hence liftable, is more subtle. We argue that existence of an anomaly cancellation mechanism is a necessary condition for liftability. In addition, the anomaly cancellation and the CS couplings in six and five dimensions respectively determine the central charges of string-like BPS objects that cannot be consistently decoupled from gravity, a.k.a. supergravity strings. Following the completeness conjecture and requiring that their worldsheet theory is unitary imposes bounds on the admissible theories. We argue that for the anomaly-free six-dimensional theories it is more advantageous to study the unitarity constraints obtained after reduction to five dimensions. In general these are slightly more stringent and can be cast in a more geometric form, highly reminiscent of the Kodaira positivity condition (KPC). Indeed, for the F-theoretic models which have an underlying Calabi-Yau threefold these can be directly compared. The unitarity constraints (UC) are in general weaker than KPC, and maybe useful in understanding the consistent models without F-theoretic realisation. We catalogue the cases when $\mathrm{UC}$ is more restrictive than $\mathrm{KPC}$, hinting at more refined hidden structure in elliptic Calabi-Yau threefolds with certain singularity structure.

Keywords: Anomalies in Field and String Theories, F-Theory, Superstring Vacua

ArXIV EPRINT: 2106.14912 


\section{Contents}

1 Introduction and discussion $\quad 1$

2 Anomalies in compactification and local counterterms 5

2.1 Compactification of $4 \mathrm{~d}$ anomalous QFT on $\mathbb{R}^{3} \times S^{1}$ : general considerations $\quad 5$

2.2 Anomalous Chern-Simons terms 7

2.3 GS mechanism and local counterterms 8

2.4 Liftable theories 9

3 BPS strings in six and five dimensions $\quad \mathbf{1 0}$

$\begin{array}{lll}3.1 & \text { BPS stings in six and five-dimensional theories with } 8 \text { supercharges } & 11\end{array}$

$\begin{array}{lll}3.2 & 5 \mathrm{~d} \text { strings from } 6 \mathrm{~d} \text { geometry } & 14\end{array}$

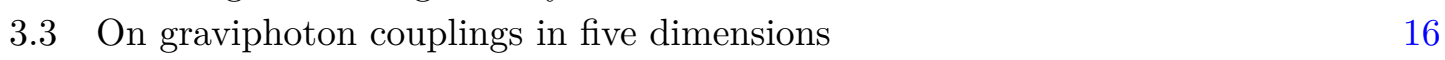

4 Five-dimensional view on the unitarity condition $\quad 18$

$\begin{array}{ll}4.1 & \text { One loop Chern-Simons couplings } 20\end{array}$

4.2 Unitarity condition for linear BPS strings 22

$\begin{array}{lll}4.3 & \text { Charges of supergravity strings } & 24\end{array}$

5 Unitarity condition as a weak Kodaira positivity condition $\quad 27$

$\begin{array}{lll}5.1 & \text { Rewriting the unitarity condition } & 28\end{array}$

5.2 Comparing KPC and UC 30

$\begin{array}{lll}\text { 5.2.1 Ample divisor } D & 32\end{array}$

$\begin{array}{lll}5.2 .2 & \text { General nef divisor } D & 35\end{array}$

5.2.3 Special cases where UC is stronger than KPC 37

$\begin{array}{lll}5.2 .4 & \text { Examples } & 38\end{array}$

$\begin{array}{ll}\text { A Reduced anomalies and Bardeen-Zumino counterterms } & 40\end{array}$

B Alternative proof that strings with $Q \cdot Q>0$ and $Q \cdot b_{i}>0$ are super-

gravity strings $\quad 43$

\section{Introduction and discussion}

A large class of odd-dimensional theories can be obtained by circle compactification of a chiral theory one dimension higher. This paper is about the way the consistency conditions of these chiral theories manifest themselves in the lower dimensional theories.

In this context, the first question about a three- or five-dimensional theory (with or without gravity) is how to determine whether the theory does indeed have a higher dimensional origin and hence can be lifted one dimension higher (we will call such theories 
"liftable"). For M-theory compactifications on Calabi-Yau three- or fourfolds, a liftability condition is well-known and captured by the structure of the internal manifold. When the CY in question is elliptically fibered, the M-theory compactification is dual to a circle reduction of F-theory on the same elliptically-fibered CY [1]. Of course such strict duality holds for smooth CY manifolds where all the gauge fields in the effective theory are Abelian. If the elliptic CY on which F-theory is compactified is singular, the even-dimensional theory has a non-Abelian gauge sector. Compactifying with Wilson lines amounts to at least partial desingularisation, and generically the elliptic structure is lost $[2,3]$. Hence, the elliptic fibration of the internal CY manifold $X$ is a necessary condition only for being able to formulate the even-dimensional theory as $\mathrm{F}$-theory on $X$, and it is a sufficient condition for being liftable for M-theory on $X$.

For concreteness, let us take $X$ to be a CY threefold, and recall that M-theory on $X$ in addition to the gravity multiplet, comprising graviton, gravitino and a vector field, has hyper and vector multiplets. All vector fields arise from the three-form field of M-theory expanded in $H^{1,1}(X, \mathbb{R})[4,5]$. The elliptic structure simultaneously ensures two different conditions. Due the to presence of at least a single two-form in $H^{1,1}(X, \mathbb{Z})$ without triple self-intersection, at least one of these vectors does not have a Chern-Simons cubic selfcoupling in five dimensions. Hence it can be dualised to a two-form [6]. Existence of such a form is a necessary condition imposed by six-dimensional supersymmetry. Much more non-trivially the ellipticity of $X$ ensures that the six-dimensional minimally supersymmetric theory obtained from F-theory on $X$ is anomaly-free. ${ }^{1}$ The anomalies (in even-dimensional theories) and the Chern-Simons couplings (in odd dimensions) are going to be central to our general discussion.

One should suspect that something interesting happens when a chiral theory with a GS term is put on a circle. By construction, the GS couplings are not invariant under the gauge symmetry and/or diffeomorphisms on spacetime $M_{2 n}$. Upon a general dimensional reduction on a circle they will continue being non-invariant. The (factorised) anomaly which these terms are supposed to cancel is due to the presence of chiral fields in the spectrum which don't exist in the odd-dimensional theory on $M_{2 n-1}$. The non-invariant part of the reduced GS terms is now cancelled by explicitly local non-invariant one-loop couplings generated by integrating out massive KK modes of the chiral fields on $M_{2 n-1} \times$ $S^{1}$ in the loop. In a series of papers [12-16] it was shown how the even dimensional conditions for a factorised anomaly, and hence for existence of GS terms, translate into correct coefficients for these new non-invariant terms needed for the cancellation. The calculations that have appeared so far are based on a specific regularisation scheme. Here we advocate a somewhat different point of view based on a more abstract argument. It can be shown how an anomaly reduced on a circle can always be written as a variation of a local term. Hence it can be fixed by an addition of a local counterterm. Schematically

\footnotetext{
${ }^{1}$ In F-theory constructions, the anomaly polynomial should be automatically sum-factorisable, and the necessary Green-Schwarz (GS) terms are induced in the reduction [7,8]. The coefficients appearing in these terms satisfy certain integrality properties, which from the effective theory point of view are seen as necessary for global anomaly cancellation [9]. A review of much of the needed background material on six-dimensional theories and F-theory can be found in [10] and in [11] respectively.
} 
this can written as:

$$
\int_{M_{2 n-1} \times S^{1}} I_{2 n}^{1}(\epsilon, \hat{\mathcal{A}}, \hat{\mathcal{F}})=\delta_{\epsilon} \int_{M_{2 n-1}} \Phi \cdot X(\mathcal{A}, \mathcal{F})+\ldots
$$

Here $\hat{\mathcal{A}} / \mathcal{A}$ and $\hat{\mathcal{F}} / \mathcal{F}$ are fields and their curvatures in $2 n$ and $2 n-1$ dimensions respecitvely, $\epsilon$ is the variation (gauge or diffeomorphism) parameter, $\Phi$ is the Wilson line along the circle, and the $\cdot$ here implies a trace over group indices. As we shall explain, $X(\mathcal{A}, \mathcal{F})$ is derived from the Bardeen-Zumino polynomial [17]. For gauge theories in a trivial gravity background, when the gauge group $G$ is preserved by the reduction, the equality is exact. When the gauge group is broken or in the case of diffeomorphism (where $\operatorname{Diff}\left(M_{2 n}\right)$ is necessarily reduced to $\left.\operatorname{Diff}\left(M_{2 n-1}\right)\right), \mathcal{A}$ and $\Phi$ are in the unbroken group, there are correction terms denoted by ellipsis.

In $2 n-1$ dimensions the non-invariance in (1.1) can always be cancelled by a local counterterm $-\Phi \cdot X$, which can never be lifted to $d=2 n$. However, it may be possible to add a different counterterm which cancels this non-invariance, which then can be lifted to the chiral theory. This is only possible when the latter admits a GS coupling. Hence the anomalies present an obstruction to liftability, and existence of a cancellation mechanism is a necessary condition for liftability. It should be interesting to find sufficient criteria.

There are other interesting questions that can be asked in this context that are beyond the scope of our paper. For the gauge anomaly reduction we have only considered the situation when the gauge group $G$ is the same before and after reduction. The argument can be augmented to include generic Wilson lines. For the gravitational theories on the contrary the reduction of the structure group is unavoidable. The proof of formula (1.1) for the gauge theory without Wilson lines in a trivial gravitational background as well as a sketchy discussion for gravitational anomalies can be found in appendix A. Finally, when starting from the lower odd-dimensional theory the question of lifting should be framed in terms of a more general obstruction theory, something that can hopefully be done in the near future.

Local anomaly cancellation is not the only consistency condition one can impose on six-dimensional minimal supergravities. A more recently developed criterion is based on the completeness conjecture of the spectrum of the charged BPS objects in supergravity theories. In six dimensions, there are string-like BPS objects that cannot be consistently decoupled from gravity (we shall follow [18, 19] and call them "supergravity strings"), provided certain conditions on their charges are satisfied. These will be spelled out in section 4. These BPS strings support two-dimensional $(0,4)$ superconformal theories on the worldsheet, whose central charges are completely fixed by the bulk anomaly cancellation, i.e. the coefficients of different couplings in GS terms. They couple to the gauge fields in the bulk for gauge group $G=\prod_{i} G_{i}$, and hence the unitarity of the worldsheet theory requires that the total central charge associated with the current algebras of $G_{i}$ is not larger than the left moving central charge:

$$
\sum_{i} c\left(G_{i}\right) \leq c_{L}
$$

The consequences of this bound for six-dimensional theories have been analysed in [18]. In contrast, the five-dimensional theories, even those that are obtained from a circle reduction, 
have a different way of packaging the information, and the expression for the $(0,4)$ central charges is rather different. We find that the five-dimensional view on the supergravity strings is somewhat more convenient and leads to constraints that are slightly more stringent. Interestingly, for the theories that come from a circle reduction the constraints are still associated with the (reduced) six-dimensional supergravity strings rather than strings carrying KK charges. ${ }^{2}$

Of course, it is natural to compare any ostensibly consistent minimally supersymmetric theory in six dimensions to F-theory constructions. In addition to the requirements imposed by physical considerations, these are subject to additional constraints that are associated with the geometry of elliptic fibrations. These constraints can be formulated either in terms of the data of the effective theory or in geometrical language. In the F-theory picture, the non-Abelian gauge groups $G_{i}$ arise from D7-branes wrapping singular gauge divisors $S_{i}$ in the base manifold $B$. The CY condition, i.e. the triviality of the canonical bundle of the elliptic fibration, relates $K$, the canonical divisor of $B$, to the locus of singular fibers. In addition the Kodaira positivity condition (KPC) states that a residual divisor $Y=-12 K-\sum_{i} x_{i} S_{i}$ should be effective. The coefficients $x_{i}$ are given by the vanishing order of the discriminant on $S_{i}$. These can be found in table 1 in section 5 . In particular this means the non-negativity of the intersection

$$
D \cdot\left(-12 K-\sum_{i} x_{i} S_{i}\right)=D \cdot Y \geq 0
$$

for any nef divisor $D$ (nef divisors, by definition, intersect every effective divisor nonnegatively). The supergravity strings in F-theory models originate from D3-branes wrapping $D$.

The unitarity conditions (UC) are formulated directly in terms of the data of the effective theory. Assuming that there is an underlying elliptic $\mathrm{CY}_{3}$ the five-dimensional UC that we derive here can be geometrised, and cast as bound on intersection forms with any nef divisor $D$. It can also be reformulated as an extra constraint on the residual divisor $Y$ :

$$
D \cdot Y \geq 3-\sum_{i}\left(x_{i}-y_{i}\right) D \cdot S_{i}
$$

where $y_{i}=\frac{\operatorname{dim} G^{i}}{1+h_{i}^{\vee}}$ and $h_{i}^{\vee}$ is the dual Coxeter number of $G_{i}$. Comparative values of $x_{i}$ and $y_{i}$ (as we shall see for any group $G_{i}, x_{i}-y_{i} \geq 1$ ) and the details of the analysis of the condition (1.4) can be found in section 5.2. A word of caution is due. This is the strongest form of the unitarity constraint, where the value of the coefficients $y_{i}$ has been computed under the assumption that $D \cdot S_{i}=1$ holds. In the vast majority of cases this bound is automatically satisfied if (1.3) holds. If it is violated, the validity of $D \cdot S_{i}=1$ needs to be checked before concluding that UC indeed imposes additional constraints on the residual divisor $Y .{ }^{3}$ In section 5.2.3 we catalogue all the cases where UC imposes extra constraints.

\footnotetext{
${ }^{2}$ Based on the anomaly inflow and local counterterms in the bulk, we can see a mismatch of BPS string spectrum in $6 \mathrm{~d}$ and $5 \mathrm{~d}$ supergravities, as discussed in section 3. As we shall argue this is explained by noticing that the $5 \mathrm{~d}$ BPS strings, carrying KK charges, are lifted to certain geometric background (TaubNUT space) that preserves half of the supersymmetry rather that BPS strings in $6 \mathrm{~d}$ supergravity.

${ }^{3}$ We have found examples where (1.4) fails, but so does the condition $D \cdot S_{i}=1$.
} 
Notice that an example where the implications of six-dimensional UC were stronger than those imposed by KPC was already presented in [18]. We find that in generic situations $5 \mathrm{~d} \mathrm{UC}$ is more constraining than $6 \mathrm{~d} \mathrm{UC}$ and has the advantage of being cast in a form directly comparable to KPC. In general it is less constraining than KPC, and hence can be useful in delineating the boundaries of the region between the six-dimensional F-theory models and the swampland (which is likely to contain a finite number of theories [20]). The fact that it does in special situations impose additional constraints allows for the intriguing possibility of finding more refined structure in elliptic $\mathrm{CY}_{3}$ with certain singularity structures. Both cases would deserve further study.

The rest of the paper is organised as follows. In section 2 we review the one-loop calculation showing how the $4 \mathrm{~d}$ anomaly reappears as Chern-Simons-like terms in $3 \mathrm{~d}$ effective theory and discuss how to understand (perturbative) anomalies in compactifications from the point of view of local counterterms. We then explore the possibility of moving in the other direction and try to interpret anomalies as obstructions of the liftability problem. All of this is done in a trivial gravitational background while the rest of the paper is set in supergravity. Details of the comparison between the 6d and 5d BPS spectra are discussed in section 3. In section 4 , we present a five-dimensional view on the unitarity constraints. In section 5, we compare the five-dimensional unitarity condition with Kodaira positivity condition and find that it is weaker in general cases. We also catalogue the special cases where the unitarity condition is stronger and may impose finer conditions on elliptic $\mathrm{CY}_{3}$.

\section{Anomalies in compactification and local counterterms}

In this section we discuss theories in a flat gravitational background. We discuss the relation between theories in even and odd dimensions if the former have anomalies. Related to this, we also address the question about the liftability of a given theory. See [12-16] for earlier discussions.

\subsection{Compactification of $4 \mathrm{~d}$ anomalous QFT on $\mathbb{R}^{3} \times S^{1}$ : general considerations}

Assume we have a $4 \mathrm{~d}_{\mathrm{QFT}}^{4}$ with global flavour symmetry $G_{F}$. The anomaly is captured by the anomaly polynomial $I_{6}(A, F)$ where $F$ is the field strength of a background gauge field $A$ which gauges $G_{F}$. Then anomaly is the non-invariance of the partition function in the presence $A$,

$$
Z\left[A^{\epsilon}\right]=\exp \left(2 \pi i \int_{M_{4}} I_{4}^{(1)}(\epsilon, A)\right) Z[A]
$$

where $\epsilon$ is the parameter of gauge transformations and $I_{4}^{(1)}$ is obtained from $I_{6}$ by the descent procedure.

For example consider $G_{F}=\mathrm{U}(1)$. In this case $I_{4}^{(1)}=N \epsilon F \wedge F$ where the normalisation depends on the field content. For instance, for a positive chirality fermion with flavour charge $q, N=\frac{q^{3}}{6(2 \pi)^{3}}$. We now consider this theory on $M_{4}=M_{3} \times S^{1}$. If we take the

\footnotetext{
${ }^{4}$ The restriction to $d=4$ is for simplicity of presentation. We will generalize to any even dimension below.
} 
components of $A$ along $M_{3}$ and the gauge parameter $\epsilon$ to be independent of the coordinate along $S^{1}$ and if we define $\phi=\int_{S^{1}} A_{4} d x^{4}$, then (2.1) becomes

$$
Z\left[A^{\epsilon}, \phi^{\epsilon}\right]=\exp \left(2 \pi i \cdot 2 N \int_{M^{3}} \epsilon d \phi \wedge d A\right) Z[A, \phi]
$$

$A$ is now the gauge field on $M_{3}, \phi$ is a scalar and

$$
A^{\epsilon}=A+d \epsilon, \quad \phi^{\epsilon}=\phi
$$

We now consider this from the point of view of a three-dimensional QFT which was obtained by compactification on a circle, whose radius is taken to zero. While in the four-dimensional parent theory the anomalous behaviour of the partition function cannot arise from the variation of a local term - otherwise the anomaly could be removed by adding a local counterterm - in the compactified theory it arises as the variation of ${ }^{5}$

$$
\mathcal{L}_{\mathrm{ct}}=-2 \pi i \cdot 2 N \phi A F
$$

whose variation produces the anomaly reduced on the circle in the presence of the Wilson line $\phi(x)$. This is the unique term with this property if we require it to be local and to depend only on the background fields.

As long as the $\mathrm{U}(1)$ symmetry is not to a gauge symmetry, this term causes no problem. It just incarnates the 't Hooft anomaly viewed from the compactified 3d QFT. However, if we gauge this anomalous $\mathrm{U}(1)$, which means $A$ and $\phi$ become dynamical fields of the QFT, this term will break the gauge symmetry explicitly. This indicates that the gauge anomaly of the $4 \mathrm{~d}$ theory will reappear as anomalous Chern-Simons terms in the $3 \mathrm{~d}$ effective theory. This argument generalizes to the non-Abelian case and also to higher dimensions.

In fact one can proof a general result. We start from the consistent anomaly $I_{2 n}^{1}(\epsilon, A, F)$ of a $2 n$-dimensional theory, as derived from the anomaly polynomial $I_{2 n+2}(A, F)$ via the descent procedure. If we compactify on a circle and turn on a Wilson line $\phi$ along the circle, the following is true:

$$
\int_{M_{2 n-1} \times S^{1}} I_{2 n}^{1}(\epsilon, \hat{A}, \hat{F})=\delta_{\epsilon} \int_{M_{2 n-1}} \phi \cdot X(A, F)
$$

$X$ is the Bardeen-Zumino polynomial

$$
X(A, F)=\frac{\partial}{\partial F} I_{2 n+1}^{0}(A, F)
$$

and the dot implies a trace over group indices. Here $\hat{A}=A+\varphi d y$ where $A$ is a one-form on $M_{2 n-1}, y$ is the coordinate along $S^{1}$ and $\int_{S^{1}} \varphi d y=\phi$. We have assumed that the gravity background is trivial. It straightforwardly generalizes if there are several gauge group factor, each with its own Wilson line.

What this result, whose proof will be given in appendix A, shows is that the compactified anomaly can always be written as the variation of a local term, i.e. it can be removed

\footnotetext{
${ }^{5}$ This term can also be derived as the compactification of the non-local term in the four-dimensional generating functional, whose variation gives rise to the anomaly.
} 
by adding an appropriate counterterm. This is, of course, no surprise, given the fact that odd-dimensional theories have no chiral anomalies, but it might be convenient to have a general expression.

A simple example is to start with $I_{6}=\operatorname{tr} F^{3}$ from which one obtains, via descent, $I_{5}^{0}=$ $\operatorname{tr}\left(A F^{2}-\frac{1}{2} A^{3} F+\frac{1}{10} A^{5}\right)$ and in $I_{4}^{1}=\operatorname{tr} \epsilon\left(A d A+\frac{1}{2} A^{3}\right)$. Then, up to a total derivative in $d=3$,

$$
\int_{S^{1}} I_{4}^{1}(\epsilon, A, F)=\delta_{\epsilon} \operatorname{tr}\left(\phi\left(F A+A F-\frac{1}{2} A^{3}\right)\right)
$$

Restricted to the Abelian case, this agrees with what we found before.

\subsection{Anomalous Chern-Simons terms}

We now want to discuss how (2.4) can be obtained from the three dimensional theory. It is well known that in three dimensions ${ }^{6}$ massive fermions induce one-loop exact Chern-Simons terms which, for a collection of $\mathrm{U}(1)$ factors is of the form

$$
\mathcal{L}_{\mathrm{cs}}=\frac{i}{8 \pi} k_{a b} \epsilon^{i j k} A_{i}^{a} \partial_{j} A_{k}^{b}
$$

The level $k_{a b}$ of the CS interaction is obtained from the parity odd part of the two-point function

$$
\left\langle j_{a}^{i}(q) j_{b}^{j}(-q)\right\rangle=\frac{1}{4 \pi} k_{a b} \epsilon^{i j k} q_{k}+\ldots
$$

It is equally well known that when compactifying from four to three dimensions, massless $4 \mathrm{~d}$ fermions give rise to an infinite tower of massive Kaluza-Klein states, where the sign of the mass depends on the $4 \mathrm{~d}$ chirality. To obtain the Chern-Simons level one needs to sum the contributions of all the KK modes. For a constant diagonal $A_{4}$ background this calculation was done in [12] whose results we summarize here. The non-zero background has two effects: it breaks the gauge group $G$ to $\mathrm{U}(1)^{\operatorname{rank}(G)}$ and shifts the KK masses of the $4 \mathrm{~d}$ fermions which transform in a non-trivial representation $\mathcal{R}$ of $G$. One finds

$$
\begin{aligned}
k_{a b} & =\sum_{\mathcal{R}} \operatorname{tr}_{\mathcal{R}} T^{a} T^{b} \sum_{n \in \mathbb{Z}} \operatorname{sign}\left(\frac{n}{L}+\frac{\phi}{2 \pi L}\right) \\
& =\sum_{\mathcal{R}}-\operatorname{tr}_{\mathcal{R}}\left(\left\{T^{a}, T^{b}\right\} T^{c}\right) \phi^{c}+\operatorname{tr}_{\mathcal{R}}\left(T^{a} T^{b} \operatorname{sign} \phi\right)
\end{aligned}
$$

where the sum has been done in $\zeta$-function regularisation. $T^{a}$ and $T^{b}$ are generators in the unbroken Abelian subgroup, i.e. in the Cartan subalgebra of $G$ and the sum is over all representations of (left handed) fermions under the gauge group $G$. $\phi=2 \pi L A_{4}$ is the Wilson line and $\left|\phi^{c}\right|<\pi$ was assumed.

Of course this result, which was computed in a gauge invariant three-dimensional theory, is gauge invariant, but it contains the information whether the $4 \mathrm{~d}$ theory was consistent. Indeed, the first part only vanishes if the Wilson line belongs to an anomaly free symmetry group, i.e. if the contributions to the anomaly of the $4 \mathrm{~d}$ theory from the various fermions cancel. If this part does not vanish and if the symmetry was a gauge symmetry, the four-dimensional theory was inconsistent, unless the anomaly can be cancelled via the

\footnotetext{
${ }^{6} \mathrm{~A}$ similar discussion is possible in any odd dimension in e.g. in $d=5$ other fields than spin $1 / 2$ fermions contribute; for details see [13].
} 
Green-Schwarz mechanism. This will be further discussed below. Here the inconsistency can be seen that for generic values of $\phi$, the CS term would not be invariant under large gauge transformations.

The second part of (2.10) has two contributions. One combines with the first part and the other arises from the piece in $\operatorname{sign}\left(\phi_{\mathcal{R}}\right)$ which is proportional to the unit matrix. This part is generically non-zero, even for a good $4 \mathrm{~d}$ theory. ${ }^{7}$

The calculation of the Chern-Simons level, for the case $G=\mathrm{U}(1)$, was reconsidered in [15], with the aim of reproducing the anomalous CS term whose variation reproduces the compactified anomaly. Here the starting point was the regularised theory in $d=$ 4. One then has to include the KK modes of the Pauli-Villars regulator fields. The introduction of the PV fields leads to the anomaly in $d=4$ and their symmetry breaking effect trickles down to the compactified theory. To obtain a result which is not invariant even under infinitesimal gauge transformations, one needs to include fluctuations $\delta \phi(x)$ around a constant background value $\bar{\phi}$ and compute also the three-point function $\left\langle j^{i} j^{j} j^{4}\right\rangle$, where the coupling is $\delta \phi j^{4}$. The two-point function in a constant background reproduces the previous result, up to its normalisation. The three-point function leads to the gaugevariant CS term (2.4) with $\phi=\delta \phi(x)$. A similar calculation in the set-up of [12] would give zero contribution from the three-point function.

\subsection{GS mechanism and local counterterms}

We have just discussed how the compactification of anomalous theories gives rise to ChernSimons terms with field dependent CS level. However it may happen that while the fermion content of the original, i.e. the uncompactified theory, is anomalous, their anomaly can be cancelled via the Green-Schwarz mechanism. This requires that the anomaly polynomial has a factorised form and that there are other fields present with inhomogeneous transformation under the gauge symmetry.

As an explicit simple example consider a factorised anomaly polynomial of the form ${ }^{8}$

$$
I_{6}=f_{A} \wedge X_{4}(f)
$$

where $f_{A}$ is the field strength of a $\mathrm{U}(1)$ factor and $f$ of a non-Abelian gauge field and $X_{4}=\operatorname{tr}\left(f^{2}\right)$. At this point, there are different ways to proceed with the descent. We can shift the anomaly into the non-Abelian or to the Abelian gauge group or a combination of both. If we put the anomaly entirely into the Abelian gauge symmetry, it is $I_{4}^{1}=\epsilon X_{4}(f)$ where $\delta a_{A}=d \epsilon$. This anomaly can be cancelled by the Green-Schwarz mechanism if the theory contains a scalar field $\varphi$ with an inhomogeneous transformation under $\mathrm{U}(1)$ gauge transformations $\delta \varphi=\epsilon$. Then adding the local term ${ }^{9}$

$$
\mathcal{L}_{\mathrm{GS}}=-\varphi X_{4}(f)
$$

to the Lagrangian, the anomaly is cancelled.

\footnotetext{
${ }^{7}$ It has the structure of a U(1)-G-G anomaly where the fermions in representation $\mathcal{R}$ of $G$ are given $\mathrm{U}(1)$ charge $\operatorname{tr}_{\mathcal{R}}(\operatorname{sign} \phi) / \operatorname{dim}(\mathcal{R})$.

${ }^{8}$ We use small letters $a$ and $f$ for dynamical gauge fields as compared to capital letters for background fields.

${ }^{9}$ It is assumed that all other terms in the action contain $\varphi$ only through its field strength $d \varphi$.
} 
As discussed before, compactifying this theory on a circle leads to the one-loop generated term

$$
\mathcal{L}_{\mathrm{CS}}^{3 \mathrm{~d}}=-2 a_{A} \operatorname{tr}(\phi f)
$$

where $\phi$ is the non-Abelian Wilson line. The compactification of the GS counterterm gives

$$
\mathcal{L}_{\mathrm{GS}}^{3 \mathrm{~d}}=2 d \varphi \operatorname{tr}(f \phi)
$$

such that their sum is gauge invariant. This is how the GS mechanism works in the compactified theory.

More generally, if an anomalous theory in four dimensions is compactified on a circle in the presence of a Wilson line, the anomaly will manifest itself in three dimensions as the non-invariance of terms in the action which are generated by fermionic KK modes. These terms can also be obtained by representing the anomaly, compactified to $3 \mathrm{~d}$, as the variation of a local term. The non-invariance of the action can be trivially removed by adding the negative of these terms as local counterterms. If the anomaly of the $4 \mathrm{~d}$ theory can be cancelled by a GS term, the same mechanism also works in 3d. This generalizes to higher dimensions.

\subsection{Liftable theories}

The space of $(2 n-1)$-dimensional theories has a subspace which consists $2 n$-dimensional theories compactified on a circle. We can then turn the question around and ask which theories in $d=2 n-1$ dimensions can be lifted to a consistent theory in one dimension higher, i.e. whether it is in this subspace. More precisely, we call a theory liftable, if we can find a consistent theory in one dimension higher whose compactification on a circle leads to the theory we started with, where we have to take into account the effect of the towers of Kaluza-Klein modes, as we have discussed before. This question is meaningful even for non-Lagrangian theories, but it is easier if a Lagrangian description exists. But even in this case we do not know the complete set of sufficient conditions for non-liftability, but some are obvious.

One is based on representations of the relevant symmetry groups. For instance a pure gauge theory in $d=3$ cannot be lifted to $d=4$ as vectors in $d=3$ do not lift to vectors in $d=4$. We need to add at least some massless scalars in the adjoint of the gauge group in $d=3$. Furthermore, the reconstruction of the $4 \mathrm{~d}$ spectrum from the $3 \mathrm{~d}$ spectrum is not unique, because e.g. the information about chirality in $4 \mathrm{~d}$ is lost when one reduces to 3d. An example where this simple representation theoretic problem can be avoided are $\mathcal{N}=2$ supersymmetric theories in $3 \mathrm{~d}$. In this case, all gauge and matter multiplets can be obtained from $\mathcal{N}=1$ multiplets in $d=4$ by dimensional reduction. For instance, vector multiplets in $d=4$ reduce to vector multiplets on $d=3$, and likewise for chiral and antichiral multiplets. It follows that $\mathcal{N}=2 \mathrm{SQCD}$ with gauge group $\mathrm{SU}\left(N_{C}\right)$ and $N_{f}$ massless chiral multiplets in the fundamental representation cannot be lifted to $d=4$.

A less trivial example related to liftability is $\mathcal{N}=2$ SQED in $d=3$ with a CS-term at level $k$, with $N_{f}$ pairs of chiral multiples $q_{i}, \tilde{q}_{i}$ and no superpotential. This theory was studied in $[21,22]$. The chiral multiplets have charge $(+1,-1)$. The theory also has a 
global symmetry $\mathrm{U}(1)_{A}$ under which the chiral multiplets have charge $(+1,+1)$. When lifted to four dimensions this symmetry is broken by an ABJ anomaly. There is also an (unbroken) $\mathrm{U}(1)_{\mathcal{R}}$ symmetry. The theory flows in the infrared to an interacting SCFT. As was shown in [23] (and reviewed in [24]), at the IR fixed point, the $\mathrm{U}(1)_{\mathcal{R}}^{\mathrm{IR}}$ symmetry is a mixture of $\mathrm{U}(1)_{\mathcal{R}}^{\mathrm{UV}}$ and $\mathrm{U}(1)_{A}$. This is, however, not a symmetry in $d=4$ due to the anomaly. This seems to mean that $\mathcal{N}=2 \mathrm{SQED}$ is not liftable. However, such a $\mathrm{U}(1)_{A}$ may be an emergent symmetry in the $3 \mathrm{~d}$ theory after compactification. If this happens the ABJ anomaly may not be able to serve as a decisive obstruction.

The general principle underlying liftability we discussed here is as follows: given a UV theory in $4 \mathrm{~d}$, we compactify on a circle with radius $r$. At high energies it looks fourdimensional but at low energies it flows to an effectively three-dimensional theory in the IR. Alternatively we compactify the $4 \mathrm{~d}$ UV theory on this circle and integrate out all Kaluza-Klein modes to arrive at a $3 \mathrm{~d}$ UV theory. We then let this flow to the IR. The two IR theories should agree if the $3 \mathrm{~d}$ UV theory is liftable to the given $4 \mathrm{~d}$ UV theory (the energy scales should be well separated as $\Lambda_{\mathrm{UV}}^{4 d} \gg \frac{1}{r} \gg \Lambda_{\mathrm{UV}}^{3 d} \gg \Lambda_{\mathrm{IR}}^{3 d}$ ).

We end this discussion with two final remarks on obstructions of liftability. One is the possibility that two $3 \mathrm{~d}$ UV theories might flow to two $3 \mathrm{~d}$ SCFTs in the IR which are dual to each other. Could it be that one of the UV theories is liftable while the other is not? The second is, could some obstruction to liftability be derived by noticing that the $3 \mathrm{~d} \mathcal{N}=2$ SUSY algebra admits a non-trivial central extension, while $4 \mathrm{~d} \mathcal{N}=1$ SUSY algebra does not? We hope to further investigate these questions in the future.

This notion of liftability is also compatible with the $6 \mathrm{~d}$ vs $5 \mathrm{~d}$ supergravity theories which we will discuss in the rest of the paper. If we consider $\mathrm{F}$ theory an elliptic $\mathrm{CY}_{3}$ and compactify on a circle to five dimensions or M theory on the same elliptic CY, they should both flow at low energies to the same 5 d supergravity theory.

\section{BPS strings in six and five dimensions}

We should now turn to supergravity theories and also switch dimensions. As we shall see there are some common features with the previous discussion. When a pair of theories is related by a circle reduction (in general with Wilson lines) the anomaly cancellation and the gauge and diffeomorphism invariance of Chern-Simons-like couplings in five dimensions is intimately related to the anomaly cancellation of the six-dimensional parent theory. As we argued, anomaly cancellation in six dimensions is a necessary condition for liftability.

Six and five-dimensional theories - even those related by a simple circle reduction have a rather different way of packaging geometric information. For example, for reductions of $\mathrm{F}$ and $\mathrm{M}$ theory on elliptic Calabi-Yau manifolds, the trilinear couplings of the former correspond to only a part of the intersection form of the CY manifold (where one of the twoforms is necessarily the pull-back from the base of the elliptic fibration), while the latter sees the entire intersection form. In a similar way, we shall argue that five-dimensional theories offer a better (and more geometric) view on the consistency of six dimensional theories (after compactifying on a circle). 
In this section, we study the spectrums of BPS strings in $6 \mathrm{~d}$ and $5 \mathrm{~d}$ minimal supergravity (eight supercharges) and point out some of the differences between them. Then we offer one way to relate the BPS strings in $6 \mathrm{~d}$ and their counterpart in $d=5$ after compactification on a circle.

\subsection{BPS stings in six and five-dimensional theories with 8 supercharges}

We consider six-dimensional theories with minimal $\mathcal{N}=1$ supersymmetry with $n_{T}$ tensor multiplets, Yang-Mills multiplets with a group $G=\prod_{i} G_{i}$ and hypermultiplets in different representations of the gauge group. A necessary condition for the Green-Schwarz anomaly cancellation mechanism is the sum-factorisation of $6 \mathrm{~d} \mathcal{N}=1$ anomaly polynomial:

$$
I_{8}=\frac{1}{2} \Omega_{\alpha \beta} X_{4}^{\alpha} X_{4}^{\beta}
$$

where $\alpha, \beta=0,1, \ldots n_{T}$ and $\Omega_{\alpha \beta}$ is the symmetric inner product on the space of tensors with signature $\left(1, n_{T}\right)$, and ${ }^{10}$

$$
X_{4}^{\alpha}=\frac{1}{8} a^{\alpha} \operatorname{tr} R^{2}+\sum_{i} b_{i}^{\alpha} \frac{1}{4 h_{i}^{\vee}} \operatorname{Tr}_{\mathrm{Adj}} F_{i}^{2}
$$

The vectors $a, b_{i} \in \mathbb{R}^{1, n_{T}}$ are determined by the field content of the theory. The anomaly cancellation condition ensures that all mutual inner products are integers. A GS term is added to the six-dimensional action to cancel the anomaly encoded in $I_{8}$ via the descent formalism.

In the presence of solitonic strings, which are the dyonic sources for self dual tensor fields, both the Green-Schwarz couplings and the Bianchi identities for the tensor fields are modified:

$$
d H^{\alpha}=X_{4}^{\alpha}+Q^{\alpha} \prod_{a=1}^{4} \delta\left(x^{a}\right) d x^{a},
$$

where $H^{\alpha}$ satisfy a self-duality condition. The 4-form distribution is the Poincaré dual to the string source and $Q^{\alpha}$ are string charges.

In addition to the standard lack of invariance under gauge transformations and diffeomorphisms, the GS term will lead to anomalous terms restricted to the string worldsheet $W_{2}$ in the presence of such a BPS solitonic string. They must cancel the anomaly of the worldsheet theory. One should bear in mind that in (3.3) the string source term is given in a particular representation of the Thom class $\Phi$ for $i: W_{2} \hookrightarrow M_{6}$, and in general it follows from the Thom isomorphism that the pull-back $i^{*} \Phi=\chi(N)$, where for the $\mathrm{SO}(4) \simeq$ $\mathrm{SU}(2)_{1} \times \mathrm{SU}(2)_{2}$ structure group of the normal bundle $\chi(N)=c_{2}\left(\mathrm{SU}(2)_{1}\right)-c_{2}\left(\mathrm{SU}(2)_{2}\right)$ is the Euler class of the normal bundle $N$ of the string. Using $\left.\operatorname{tr} R^{2}\right|_{T W_{2}}=-2 p_{1}\left(T W_{2}\right)-2 p_{1}(N)$ and $p_{1}(N)=-2\left(c_{2}\left(\mathrm{SU}(2)_{1}\right)+c_{2}\left(\mathrm{SU}(2)_{2}\right)\right)$, one infers that the anomaly two-form on $W_{2}$ is obtained via descent from

$$
\begin{aligned}
I_{4} & =-\Omega_{\alpha \beta} Q^{\alpha}\left(\left.X_{4}^{\beta}\left(M_{6}\right)\right|_{W_{2}}+\frac{1}{2} Q^{\beta} \chi(N)\right) \\
& =-\frac{1}{4} \Omega_{\alpha \beta} Q^{\alpha}\left(a^{\beta} p_{1}\left(T W_{2}\right)-2\left(Q^{\beta}+a^{\beta}\right) c_{2}\left(\mathrm{SU}(2)_{1}\right)+2\left(Q^{\beta}-a^{\beta}\right) c_{2}\left(\mathrm{SU}(2)_{2}\right)+\ldots\right)
\end{aligned}
$$

\footnotetext{
${ }^{10}$ Our normalisations of the curvatures $R$ and $F$ are such that they contain a factor $1 / 2 \pi$.
} 
The ellipsis stands for the pullback of the YM part in (3.2) which is not needed for the following analysis.

The theory on the worldsheet flows in the IR to a $(0,4)$ SCFT and the information about the left and right central charges as well as the level of the $\mathrm{SU}(2) \mathcal{R}$-symmetry current algebra is contained in $I_{4}$. As discussed in detail in [18], the SCFT splits into a free center of mass SCFT and an interacting SCFT. The former consists of a hypermultiplet with left and right central charges 4 and 6 , respectively. Its $\mathcal{R}$-symmetry group is not contained in the $\mathrm{SO}(4)$ from the normal bundle as the four scalars, which are neutral under the $\mathcal{R}$-symmetry, transform as a vector of $\mathrm{SO}(4)$. From the point of view of the worldsheet theory it is an accidental symmetry. The contribution of the c.o.m. part to $I_{4}$ is $-\frac{1}{12} p_{1}\left(T W_{2}\right)-c_{2}\left(\mathrm{SU}(2)_{1}\right)$. In particular it does not interfere with the $\mathcal{R}$-symmetry of the interaction part of the SCFT, which is $\mathrm{SU}(2)_{2}$. Using the $(0,4)$ relation $c_{R}=6 k_{\mathcal{R}}$ between the central charge and the level of the $\mathcal{R}$-current algebra, we can read off $c_{R}^{\text {int }}$ from the $c_{2}\left(\mathrm{SU}(2)_{2}\right)$ part of $I_{4}$ and $c_{R}^{\text {int }}-c_{L}^{\text {int }}$ from the coefficient $p_{1}\left(T W_{2}\right)$ of the gravitational anomaly. Adding the contribution of the c.o.m. part one finds

$$
\begin{aligned}
c_{L}-c_{R} & =-6 \Omega_{\alpha \beta} a^{\alpha} Q^{\beta} \equiv-6 Q \cdot a \\
c_{R} & =3 \Omega_{\alpha \beta} Q^{\alpha} Q^{\beta}-6 \Omega_{\alpha \beta} a^{\alpha} Q^{\beta}+6 \equiv 3 Q \cdot Q-6 Q \cdot a+6
\end{aligned}
$$

We have defined here an inner product denoted by - using the metric on the space of tensors $\Omega_{\alpha \beta}$.

Following $[18,19]$ we shall be interested in supergravity strings, ${ }^{11}$ whose worldsheet $\mathcal{R}$-symmetry descends from the structure (sub)group of the normal bundle. This condition restraints the values of the admissible $Q$ charges. Once such restrictions are imposed, the worldsheet SCFT should be unitary, i.e. the central charge $c_{L}$ should serve as a bound for the contribution of the left moving current algebra for $G$ at level $k$ :

$$
\sum_{i} \frac{k_{i} \cdot \operatorname{dim} G_{i}}{k_{i}+h_{i}^{\vee}} \leq c_{L}-4
$$

where for Abelian gauge factors $h^{\vee}=0$.

So far we have discussed solitonic strings in $d=6$. Most of the subsequent analysis will be five-dimensional, and we shall in particular be interested in the five-dimensional solitonic strings obtained via circle reduction, when the $S^{1}$ is transverse to the six-dimensional string. To get the anomaly formula of the resulting $(0,4)$ SCFT on the string, we simply let the normal bundle be $\mathbb{R}^{3} \times S^{1}$. To go to five dimensions, we take $c_{2}\left(\mathrm{SU}(2)_{1}\right)=c_{2}\left(\mathrm{SU}(2)_{2}\right)=$ $c_{2}(N)$, where $N$ is the $S^{2}$ normal bundle fiber inside $\mathbb{R}^{3}$. Imposing this in (3.4) leads to

$$
c_{L}=2 c_{R}=-12 \Omega_{\alpha \beta} a^{\alpha} Q^{\beta} \equiv-12 Q \cdot a
$$

preserving the difference $c_{L}-c_{R}$ (3.5). Such five-dimensional solitonic strings with central charges linear in $Q$ are magnetic sources for the $\mathrm{U}(1)$ gauge fields obtained from the reduction of the six-dimensional tensor fields.

\footnotetext{
${ }^{11}$ I.e. BPS strings that cannot be consistently decoupled from gravity.
} 
We now turn to the generic string sources in five-dimensional $\mathcal{N}=1$ supergravity. Such a BPS string also hosts a $(0,4) 2 \mathrm{~d}$ SCFT on its worldsheet, hence we can obtain $c_{L}, c_{R}$ for this $2 \mathrm{~d}$ SCFT via anomaly inflow caused by $5 \mathrm{~d}$ bulk Chern-Simons terms:

$$
\frac{1}{96} a_{I} A^{I} \operatorname{tr}(R \wedge R)-\frac{1}{6} C_{I J K} A^{I} \wedge F^{I} \wedge F^{J}
$$

From these Chern-Simons terms we obtain [19, 25, 26]

$$
\begin{aligned}
& c_{R}=C_{I J K} Q^{I} Q^{J} Q^{K}+\frac{1}{2} a_{I} Q^{I} \\
& c_{L}=C_{I J K} Q^{I} Q^{J} Q^{K}+a_{I} Q^{I}
\end{aligned}
$$

The index $I$ runs over all $d=5$ vectors. In 6 d language, $I=1, \ldots, n_{T}+n_{V}+1$.

The structure of central charges of $(0,4)$ SCFTs hosted on 5d BPS strings is very different from $6 \mathrm{~d}$ ones. While in general for $6 \mathrm{~d}$ strings the leading behaviour for both $c_{L}$ and $c_{R}$ is quadratic in $Q$, due the quadratic terms in the anomaly polynomial (3.1), in five dimensions it is generally cubic. Moreover, in five dimensions the anomaly inflow cannot produce central charges with quadratic scaling in $Q$.

For the vector fields originating form six-dimensional tensors, the coefficient of the gravitational coupling does not renormalise upon reduction and the triple self-intersection does not get generated. One recovers the central charges as in (3.7) linear in $Q$ and with $c_{L}=2 c_{R}$. So the conclusion would be that for the $5 \mathrm{~d}$ BPS strings from $6 \mathrm{~d}$ BPS string compactified on a transverse circle, the central charge $c_{L}, c_{R}$ on the $(0,4)$ SCFT it hosted will have vanishing cubic term (i.e. $C_{I J K} Q^{I} Q^{J} Q^{K}=0$ in (3.9)).

For the remaining $\mathrm{U}(1)$ vectors in $5 \mathrm{~d} \mathcal{N}=1$ supergravity, including the graviphoton $A^{0}$, integrating out of the massive $\mathrm{KK}$ tower in general cases generates the gravitational couplings with coefficients $a_{I}$ and the trilinear self-intersections with (non-zero) coefficients $C_{I J K}$. The central charge of these strings in general have a cubic dependance on $Q$. We shall refer to these types of BPS strings as linear(central charge with vanishing cubic term $\left.C_{I J K} Q^{I} Q^{J} Q^{K}=0\right)$ and cubic for the cases in the subsequent discussion. ${ }^{12}$

In sections 4 and 5 we shall concentrate on the linear strings, and re-examine the unitarity constraints of the six-dimensional theories from five-dimensional view-point. Given the change in the nature of $c_{L}$ in passage form six to five dimensions, the unitarity constraints, as we shall see, are different both in substance (they are in general a bit stronger) and in form (they appear to be more geometric). We did not find the cubic strings to be amendable to such analysis and to produce useful constraints. However in the remainder of this section we shall elucidate their six-dimensional origin.

We close this section with addressing two points about the central charge formula (3.9):

- We assume the $(0,4)$ superconformal algebra to be the small superconformal algebra (hence the relation $c_{R}=6 k_{\mathcal{R}}$ ) rather than the large ones. ${ }^{13}$ This is reasonable

\footnotetext{
${ }^{12}$ A little clarification is due. "Linear strings" can have trilinear dependance in the central charges which can however be set to zero by appropriate choices of the charge vector. This is the case with the self-dual string in $\mathcal{N}=1$ theory after circle reduction. As we shall see, their central charges can acquire contributions $\sim Q_{\mathrm{KK}}^{2} Q$. However $Q_{\mathrm{KK}}$ can be consistently taken to zero. The cubic strings, on the contrary, are charged with respect to vector fields that have a cubic self-coupling.

${ }^{13}$ We thank the referee for raising these points.
} 
since the large $(0,4)$ superconformal algebras have $\mathrm{SU}(2)_{1} \times \mathrm{SU}(2)_{2}$ as (part of) their $\mathcal{R}$-symmetry and exotic BPS conditions [27], which make them unlikely to be the candidates of the $(0,4)$ superconformal algebra on the BPS strings at IR.

- We assume the $\mathrm{SU}(2) \mathcal{R}$-symmetry of the $(0,4)$ superconformal algebra is associated with the normal bundle of the BPS strings. For supergravity BPS strings, which are the objects discussed in this paper, the $6 \mathrm{~d}$ bulk is always a theory of gravity and no global symmetries can appear. Therefore there is no ambiguity in identifying the $\mathcal{R}$-symmetry that come from some other $\mathrm{SU}(2)$ symmetries in the bulk.

\section{$3.2 \quad 5 d$ strings from $6 \mathrm{~d}$ geometry}

We will now argue that the five-dimensional cubic strings originate from the six-dimensional geometry $\mathbb{R}^{1,1} \times M_{\mathrm{TN}}$, i.e. when the circle on which the theory is reduced is non-trivially fibered. Moreover, every cubic string should carry some KK (magnetic)charge. As we shall see this argument is consistent with F-theoretic considerations.

We have already seen that the reduction of six-dimensional strings, which are charged under the tensor fields, yields only linear strings. Hence the cubic strings can only be charged under the vectors that come from the reduction of six-dimensional vector multiplets or under the KK vector $g_{\mu 5}$. One could wonder if there is a solitonic object (a membrane) in six dimensions that is charged under the $\mathrm{U}(1)$ fields and whose reduction yields the cubic strings. If so, the $(0,4) \mathrm{SCFT}$ on the $5 \mathrm{~d}$ string should arise from $3 \mathrm{~d} \mathcal{N}=2$ QFT on the membrane compactified on a $S^{1}$. This generally cannot produce a chiral theory in two dimensions (notice that our $2 \mathrm{~d}$ theory is obtained from a compactification of a $3 \mathrm{~d}$ theory on a circle, not via restriction to the boundary of a $3 \mathrm{~d}$ theory). Also, obviously the magnetic sources for the KK vector after circle compactification do not arise from any wrapped object in $6 \mathrm{~d}$ either as the $6 \mathrm{~d}$ theory itself does not have the KK vector.

To find the $6 \mathrm{~d}$ origin for the cubic BPS strings after circle compactification, let us recall that for five-dimensional supergravities obtained from the compactifications of M-theory on an elliptically fibered $\mathrm{CY}_{3}$, the $(0,4)$ cubic strings arise from M5 branes wrapping a smooth ample divisor. ${ }^{14}$ So let us have a closer look at ample divisors in a smooth elliptically fibered $\mathrm{CY}_{3}$ :

$$
E_{\tau} \rightarrow \mathrm{CY}_{3} \rightarrow B
$$

These can comprise the base $B$ and $\pi^{-1}\left(\Sigma_{i}\right)$, which are pullbacks of curves in the base, and an expectional divisor $X$. Hence the generic ample divisor $D$ can be written as

$$
D=a B+b \pi^{-1}(\Sigma)+X .
$$

It follows from the Nakai-Moishezon ampleness condition for $D$, which implies

$$
D \cdot D \cdot D>0 \quad \text { and } \quad D \cdot C>0
$$

\footnotetext{
${ }^{14}$ In order to see the microscopic origin on the central charge formula in terms of the zero modes of the fields on M5 one should assume that the divisor is very ample [28].
} 
for any effective curve $C$, that $a \neq 0$, i.e. any ample divisor in a smooth $\mathrm{CY}_{3}$ necessarily contains some copies of the base. Indeed, this follows immediately if we take $C$ to be the intersection of two $\pi^{-1}\left(\Sigma_{i}\right)$, and use that

$$
\pi^{-1}\left(\Sigma_{i}\right) \cdot \pi^{-1}\left(\Sigma_{j}\right) \cdot \pi^{-1}\left(\Sigma_{k}\right)=0 \quad \text { and } \quad \pi^{-1}\left(\Sigma_{i}\right) \cdot \pi^{-1}\left(\Sigma_{j}\right) \cdot X=0 .
$$

Moreover, in the M theory picture, an M5 brane wrapping the base is a magnetic source for the KK vector. So from the M/F theory points of view, the cubic string should carry some magnetic charge of the KK vectors. In general this implies that the six-dimensional counterpart of these strings should contain the KK monopole configuration, which is naturally given by Euclidean Taub-NUT geometry (see e.g. [29]):

$$
d s_{6}^{2}=-d t^{2}+d y^{2}+d s_{\mathrm{TN}}^{2}
$$

with

$$
d s_{\mathrm{TN}}^{2}=\left(1+\frac{Q R_{0}}{r}\right)\left(d r^{2}+r^{2} d \Omega_{2}\right)+R_{0}^{2}\left(1+\frac{Q R_{0}}{r}\right)^{-1}(2 d \psi+Q A)^{2}
$$

Here $Q \equiv Q_{\mathrm{KK}}$, the KK monopole charge, is a integer; we will restrict to the positive integer case for simplicity and without loss of generality. $d A=d \Omega_{2}$ is the volume element on the unit 2-sphere and $\psi \simeq \psi+2 \pi$. The TN space is a $S^{1}$ fibration over $\mathbb{R}^{3}$ (except the locus where the $S^{1}$ fiber shrinks to zero size). Far away from the origin the space is $S^{1} \times \mathbb{R}^{3}$ where the radius of the circle is $2 R_{0}$. This is the circle we want to compactify on. It shrinks to zero size at the origin at $r=0$ where the space has a $A_{Q-1}$ singularity. This is the limit of an $Q$-centered TN space where all centers coincide (here at the origin $r=0$ ).

For a fixed small distance $r=\epsilon$, we can neglect the constant in the harmonic function and the metric becomes that of an $S^{1}$ fibration over $S^{2}$ (a cyclic Lens-space)

$$
S^{1} \rightarrow S^{3} \rightarrow S^{2}
$$

It is characterised by the first Chern number of the KK vector

$$
\lim _{\epsilon \rightarrow 0} \int_{S_{\epsilon}^{2}} \frac{F^{\mathrm{KK}}}{2 \pi}=Q_{\mathrm{KK}}
$$

The argument that $5 \mathrm{~d}$ cubic strings should come from the $6 \mathrm{~d}$ theory on a Taub-NUT background after compactification on the circle fiber, can be generalised to include sixdimensional U(1) vector fields following the generalised Taub-NUT solution in [30]. These will give solitonic string-like objects which carry both KK as well as the related U(1) magnetic charges after compactification on the circle. As the Taub-NUT metric is a gravitational instanton, half of the supersymmetry is preserved by this background, just as it is expected for the string solitons with $(0,4)$ worldsheet supersymmetry.

Finally this picture also accounts qualitatively for the chirality of the theory on the string worldsheet. Given that the six-dimensional theory has a self-dual tensor field in the gravity multiplets and $n_{T}$ anti-self-dual tensors in tensor multiplets, their decomposition along the basis of self-dual and anti-self-dual $(1,1)$ forms on $M_{\mathrm{TN}}$ yield two-dimensional 
modes $b(t, y)$, where $(t, y)$ denote the coordinates along $R^{1,1}$, i.e. the string worldsheet, such that

$$
\left(\partial_{t} \mp \partial_{y}\right) b(t, y)=0
$$

Note this is only part of the spectrum and this analysis is on the $6 \mathrm{~d}$ UV side. So we cannot use this argument to determine $c_{L}$ and $c_{R}$ of the resulting $(0,4)$ SCFT individually. However the chirality of spectrum implies ' $t$ Hooft anomalies, which match between the UV and the IR. Hence the resulting solitonic string from Taub-NUT reduction should support a chiral spectrum in the IR. The more direct argument is using anomaly inflow of the compactified five-dimensional theory, as we did before.

We can consider more general configurations. Six-dimensional $\mathcal{N}=1$ supergravity theory in a (generalised) Taub-NUT background and a BPS string at the locus where the $S^{1}$ fiber shrinks to zero size (the two objects preserve the same set of supercharges), after compactification yields five-dimensional supergravity with solitonic BPS strings. Moreover, these 5d BPS strings carry magnetic charges for the U(1) gauge fields as well as the KK charge. Since upon such reduction cubic self-couplings of the $\mathrm{U}(1)$ fields are generated these string configuration will, in general, have cubic central charges.

In summary, we have argued that cubic BPS strings in $5 \mathrm{~d} \mathcal{N}=1$ supergravity obtained from minimal 6d supergravity originate from a (generalised) Taub-NUT background.

\subsection{On graviphoton couplings in five dimensions}

The claim that in five-dimensional theories, obtained via circle reduction of six-dimensional $\mathcal{N}=1$ supergravity, the cubic solitonic strings arise from non-trivial geometric backgrounds, immediately leads to the following requirement:

- Since we can always turn on a purely geometric Taub-NUT background with arbitrary KK monopole charge, there should always be a solitonic string which only carries KK magnetic charge and supports a $(0,4)$ or $(4,0)$ SCFT. ${ }^{15}$ The superconformal algebra and unitarity then require $c_{R}\left(\right.$ or $\left.c_{L}\right)=6 k_{\mathrm{SU}(2)_{\mathcal{R}}} \in \mathbb{Z}_{+}$.

To this end, it suffices to consider the Chern-Simons-like couplings to the KK vector in five dimensions. For the $S^{1}$ reduction of the Taub-NUT background, the magnetic string charged under the KK vector is at the position where the $S^{1}$ shrinks to zero size. Far away from this string, the five-dimensional physics can be derived by just putting a $(0,1)$ theory on a circle. So the corresponding Chern-Simons level can be obtained by integrating out the massive charged modes in a one-loop Feynman diagram calculation [13]. The relevant couplings are given by

$$
\mathcal{L}_{\mathrm{CS}}=-\frac{k_{0}}{6} A^{\mathrm{KK}} \wedge F^{\mathrm{KK}} \wedge F^{\mathrm{KK}}+\frac{k_{R}}{96} A^{\mathrm{KK}} \wedge \operatorname{tr} R^{2}
$$

\footnotetext{
${ }^{15}$ To determine $(0,4)$ vs $(4,0)$ is by looking at the $\mathrm{SU}(2) \mathcal{R}$-symmetry part of the anomaly polynomial which is $\pm k_{\mathcal{R}} c_{2}\left(\mathrm{SU}(2)_{\mathcal{R}}\right)$. Here $k_{\mathcal{R}}$ is the level of the $\mathrm{SU}(2)_{\mathcal{R}}$ current algebra, which unitarity requires to be positive. For the minus sign we have a $(0,4)$ and for the plus sign a $(4,0)$ SCFT on the string worldsheet.
} 
and the ensuing central charges $c_{L}$ and $c_{R}$ are obtained from the inflow arguments (for a $(0,4)$ SCFT on the string) as

$$
c_{R}=k_{0} Q_{\mathrm{KK}}^{3}+\frac{k_{R}}{2} Q_{\mathrm{KK}} \quad c_{L}=k_{0} Q_{\mathrm{KK}}^{3}+k_{R} Q_{\mathrm{KK}}
$$

where $Q_{\mathrm{KK}}$ is the KK string charge.

The coefficients $k_{0}, k_{R}$ depend on the six-dimensional field content. They have been calculated in [13]:

$$
k_{0} \stackrel{?}{=} \frac{9-n_{T}}{4}, \quad k_{R} \stackrel{?}{=} 4\left(12-n_{T}\right)
$$

where we have indicated that one should be careful to accept these results in the present context.

Indeed, for $c_{R}$ to be the central charge of a $(0,4)$ SCFT, it should be an integer divisible by 6 , as unitarity constrains the level $k_{\mathrm{SU}(2)_{\mathcal{R}}}$ of the $\mathrm{SU}(2)_{\mathcal{R}}$ current algebra to be a (positive) integer. It is easy to verify that this is not the case for general $n_{T}$ and $Q_{\mathrm{KK}}$. On the other hand, a large range of values for $n_{T}$ and $Q_{\mathrm{KK}}$ is allowed. For instance, for $\mathcal{N}=1$ theories obtained from F-theory, generically $n_{T}$ can be shifted by 1 through blowing-up or blowing-down a rational curve on the base with self-intersection number -1 . Furthermore, in a phase transition proposed in [31], we can trade one tensor multiplet for 29 hyper multiplets by blowing down an exceptional divisor on the Hirzebruch surface $F_{1}$ to get $\mathbb{P}^{2}$. And the value of $Q_{\mathrm{KK}}$ is free (except that it should be large, as will be discussed later). Furthermore, the value of $k_{0}$ in (3.21) is generically not an integer, violating the quantisation condition of the CS level discussed in [31].

We will now argue that (3.21) should be replaced by

$$
k_{0}=2\left(9-n_{T}\right), \quad k_{R}=8\left(12-n_{T}\right)
$$

which obviously solves the problems just discussed and $c_{R}$ (or $\left.c_{L}\right)=6 k_{\mathrm{SU}(2)_{\mathcal{R}}} \in 6 \mathbb{Z}_{+}$ is satisfied.

Consider the Taub-NUT metric (3.15) with a unit magnetic charge $Q_{\mathrm{KK}}=1$. It interpolates between $\mathbb{R}^{3} \times S^{1}$ for large $r$ and $\mathbb{R}^{4}$ for small $r$. There the metric on $\mathbb{R}^{4}$ is given in polar coordinates where the $S^{3}$ at fixed $r$ is parametrised by Euler angles $(\theta, \phi, \psi)$, except that we have rescaled $\psi$ such as to give it periodicity $2 \pi$ rather than $4 \pi . \theta$ and $\phi$ are coordinates on $S^{2}$, i.e. $d \Omega_{2}=d \theta^{2}+\sin ^{2} \theta d \phi^{2}$. Besides the $2 \pi$ periodicity of $\psi$, this parametrisation of $S^{3}$ is invariant under the combined transformations

$$
(\phi, \psi) \sim(\phi+2 \pi, \psi+\pi),
$$

After compactification, in the five-dimensional effective theory we can explicitly impose $\phi \sim \phi+2 \pi$, but then the condition $\psi \sim \psi+\pi$ is lost. As a result, for a consistent compactification, we need to impose these two conditions on the fields separately. For example, for a fermion field $\lambda^{6 d}(\phi, \psi)$ in this background, we require not only $\lambda^{6 d}(\phi, \psi)=$ $\lambda^{6 d}(\phi, \psi+2 \pi)$, but also $\lambda^{6 d}(\phi, \psi)=\lambda^{6 d}(\phi+2 \pi, \psi+\pi)$. In fact, fixing the magnetic KK charge $Q_{\mathrm{KK}}=1$, implies that all electric KK charges take even values. ${ }^{16}$

\footnotetext{
${ }^{16}$ Conversely, had the electric KK charge been fixed at unity, all magnetic KK charges would have to take even values to satisfy the quantisation condition as proposed in [31].
} 
Imposing that the magnetic charges take values in $\mathbb{Z}$, we need to require $\psi \sim \psi+\pi$ rather than $\psi \sim \psi+2 \pi$. Consequently the decomposition of six-dimensional fields is

$$
\lambda^{6 d}=\sum_{n} \lambda_{n}^{5 d} \exp (2 \pi i \times 2 n \psi)
$$

This means that all fields carry even electric KK charge due to the existence of a KK magnetic monopole of charge 1 . The triangle diagrams with the tower of charged KK modes in the loop, which was computed in [13] and which leads to the Chern-Simons terms (3.19), therefore has to be modified accordingly. There is an additional factor of two for each coupling to the KK gauge field. This gives (3.22).

This has a very natural counterpart in the M/F theory framework. As shown in [13], assuming (3.21) is an equality, leads to the identification of the KK vector in M-theory on a $\mathrm{CY}_{3}$ with the eleven-dimensional three-form along the shifted two-form $\operatorname{PD}(B)+\frac{1}{2} c_{1}(B)$. Here $B$ is the base of the elliptic fibration, $c_{1}(B)$ its first Chern class and $\operatorname{PD}(B)$ its Poincaré dual. The string charged under the KK vector is to be identified with an M5 wrapping the corresponding divisor $B+\frac{1}{2}\left[c_{1}(B)\right]$. But in a generic geometry, an M5 brane cannot wrap $\frac{1}{2}\left[c_{1}(B)\right]$, and the corresponding magnetic charge should be obtained by an M5 wrapping this formal divisor as a result $2 n$ times. This is exactly the requirement that the magnetic charge takes values in $2 \mathbb{Z}$. Keeping the standard quantisation $[F] \in H^{2}\left(M_{5}, \mathbb{Z}\right)$ we need to reinstate this factor of 2 elsewhere. In particular, we can identify the KK vector as the mode of the M-theory three-form along $2 \mathrm{PD}(B)+c_{1}(B)$. In particular an M5 brane can wrap the corresponding divisor $2 B+\left[c_{1}(B)\right]$ once, leading to a unit magnetic charge.

\section{Five-dimensional view on the unitarity condition}

Six-dimensional gravitational and gauge anomalies in $6 \mathrm{~d}$ minimal supersymmetric theories allow not only to read off the central charges of stringy objects with $(0,4)$ worldsheet supersymmetry, but also the level $k_{L}$ of the current algebra that couples to the left-movers. The condition that the left-moving central charge is large enough to allow for a unitary representation of the current algebra at level $k_{L}$ was used in [18] as a consistency condition of quantum gravity in order to rule out some anomaly-free $6 \mathrm{~d}$ minimal supergravity theories. We shall re-examine these constraints, for which we shall use the shorthand "unitarity conditions", from a five-dimensional perspective.

As mentioned previously, the chiral two-dimensional theories that live on the string worldsheet are $(0,4)$ (or $(4,0))$ SCFT, i.e the $\mathrm{SU}(2)_{\mathcal{R}}$ symmetry inherited from the normal bundle of this string belongs to the right-moving (left-moving) sector. Unitarity of the worldsheet theory requires that the central charges are positive. This should in particular be true for the string charged under the KK vector for which $c_{R}=6 k_{\mathcal{R}}$ (or $c_{L}=6 k_{\mathcal{R}}$ ) is given in (3.20). However a closer look at this expression seems to lead to a puzzle:

- for $n_{T} \leq 9$, the string $\operatorname{SCFT}$ has $(0,4)$ supersymmetry and $c_{R}=6 k_{\mathcal{R}}=$ $2\left(9-n_{T}\right) Q_{\mathrm{KK}}^{3}+4\left(12-n_{T}\right) Q_{\mathrm{KK}}>0$ for $Q_{\mathrm{KK}}>0$;

- for $n_{T} \geq 12$, the string has a $(4,0)$ worldsheet SCFT and $c_{L}=6 k_{\mathcal{R}}=2\left(n_{T}-9\right) Q_{\mathrm{KK}}^{3}+$ $4\left(n_{T}-12\right) Q_{\mathrm{KK}}>0$ for $Q_{\mathrm{KK}}>0$; 
- for $n_{T}=10,11$, something unpleasant happens. Take $n_{T}=10$ for example, then $2\left(9-n_{T}\right) Q_{\mathrm{KK}}^{3}+4\left(12-n_{T}\right) Q_{\mathrm{KK}}=-2 Q_{\mathrm{KK}}^{3}+8 Q_{\mathrm{KK}}$, which gives $6,0,-30$ for $Q_{\mathrm{KK}}=1,2,3$, respectively. This would seem to indicate that the string has $(0,4)$ supersymmetry for $Q_{\mathrm{KK}}=1$ and $(4,0)$ supersymmetry for $Q_{\mathrm{KK}}=3$. But if this KK monopole string indeed originates in the Taub-NUT background in six dimensions, all positive values of $Q_{\mathrm{KK}}$ should be allowed, and it is hard to imagine such changes from a change in the value of $Q_{\mathrm{KK}}$.

The puzzle is resolved by realising that our considerations of the BPS strings have implicitly assumed that $Q_{\mathrm{KK}}$ is sufficiently large. Indeed, the Taub-NUT metric (3.15) has an intrinsic scale, the radius of the compactification circle $2 R_{0}$. Therefore, the fivedimensional supergravity description can only be trusted below the energy scale $\Lambda_{5 \text { d-SUGRA }} \simeq$ $\frac{1}{2 R_{0}}$. On the other hand, the anomaly inflow calculation leading to (3.8) and (3.9) required a smeared-out version of the Bianchi identity $d F=\frac{Q}{2} d \rho(r) \wedge e_{2}$, see [25, 26] for relevant details, which involves a function $\rho(r)$ of the distance away from the string. As this bump function, which interpolates between -1 and 0 , hides UV physics which is not visible in the $5 \mathrm{~d}$ supergravity description, its radial compact support should be of the order $2 R_{0}$. On the other hand, in the $5 \mathrm{~d}$ supergravity description which we used above, the string source should be treated as a $\delta$-function in the directions transverse to its worldsheet. In other words its thickness $\delta r$ should go to zero. Using the explicit form of the TN metric (3.15), this translates into the condition

$$
\int_{0}^{\delta r} \sqrt{1+\frac{R_{0} Q_{\mathrm{KK}}}{x}} d x=2 R_{0} \quad \text { with } \quad \delta r \rightarrow 0
$$

This leads to $\delta r \sim \frac{R_{0}}{Q_{\mathrm{KK}}} \rightarrow 0$ for fixed $R_{0}$, i.e. $Q_{\mathrm{KK}}$ should be large. It is under this condition that the values of the central charge derived from bulk anomaly inflow can be trusted.

In the M-theory picture, where the string arises from an M5-brane wrapping a divisor, this translates into the very ampleness condition on the divisor [28].

The argument for large $Q_{\mathrm{KK}}$ is supported indirectly by considering specific sixdimensional theories. Take for example an anomaly free $6 \mathrm{~d}$ minimal supergravity theory with $n_{T}=10$ or $n_{T}=11, n_{v}=\operatorname{rk}(G)$ (for some suitable gauge group $G$ ) and $Q_{\mathrm{KK}}=1$. Then the value of the left-moving central charge on the KK string is $c_{L}=14$ or $c_{L}=4$ respectively, and hence the unitarity of the worldsheet SCFT would require $\operatorname{rk}(G) \leq c_{L}=14$ or $4 .{ }^{17}$ This requirement is obviously too strong. Heterotic string on K3 with 9 or 10 five-branes respectively and an $\mathrm{SU}(2)$ instanton (with instanton number 15 or 14) easily provides counterexamples to this. Given the cubic dependance of $c_{L}$ on $Q_{\mathrm{KK}}$ this requirement is easily satisfied for larger charges.

As a result, we have shown that we only have to distinguish two situations depending on the value of $n_{T}$

- $n_{T} \leq 9$, KK monopole string supports $(0,4)$ supersymmetry

- $n_{T}>9$, KK monopole supports $(4,0)$ supersymmetry.

\footnotetext{
${ }^{17}$ Note that in five dimensions couplings $\sim A^{\mathrm{KK}} \wedge F^{i} \wedge F^{j}(i, j=1, \ldots, \operatorname{rk}(G))$ are generated at one loop, and the KK string couples to the gauge sector.
} 
Note that the value of $n_{T}=9$ is somewhat special. For the F-theory models on ellipticallyfibered $\mathrm{CY}_{3} X, n_{T}=9+\chi(X) / 60$ where $\chi(X)$ is the CY Euler number. When $\chi$ vanishes, i.e. $n_{T}=9$, the effective theory has another set of hidden supersymmetries (and can be thought of as a gauged supergravity theory with 16 supercharges) [32]. Correspondingly one would expect that the solitonic supergravity string may also display extra worldsheet supersymmetry and be enhanced to $(0,8)$. If so the superconformal algebra will require $c_{R} \in 12 \mathbb{Z}$. The KK monopole strings satisfies this requirement, as one easily sees from (3.20) and (3.22) with $n_{T}=9$.

There is an immediate consequence of the large $Q_{\mathrm{KK}}$ requirement for the unitarity analysis. Due to the presence of $Q_{\mathrm{KK}}^{3}$, the left-moving central charge $c_{L}$ grows very fast, and hence does not give strong constraints. As we have argued, every cubic string in fivedimensional theories (obtained from a circle compactification of $6 \mathrm{~d}$ supergravity) carries KK charge. It being large renders a generic cubic string rather useless as far as the unitarity constraints go. Of course this is not the case for the linear strings that come from the sixdimensional supergravity strings. Hence our five-dimensional unitarity analysis will be applied to the very same objects that have been analysed in [18].

One can argue quite generally that the dimensional reduction should not be imposing any new consistency conditions (even if, as it is the case here, it can repackage these in a new and useful fashion). Although we know that sometimes IR properties can be used to constrain the possible UV completion (e.g. $c$-theorem in $2 \mathrm{~d}$, or $a$-theorem in $4 \mathrm{~d}$, or the obstructions of liftability discussed previously), this is not the case in the current $5 d / 6 d$ context. Here we know both the $6 \mathrm{~d}$ UV side and $5 \mathrm{~d}$ IR side, as well as the correspondence of the extended objects on both sides. Since the Taub-NUT background does not cause any inconsistencies on the six-dimensional UV side, no inconsistencies should be generated along the RG flow.

\subsection{One loop Chern-Simons couplings}

In this subsection we want to discuss Chern-Simons couplings which are generated in five dimensions after integrating out the massive KK modes which arise upon compactification on a circle; see also the discussion in section 2. In general Wilson lines can be switched on, and this is the case of interest for us. Before turning to it, we will briefly review the case without Wilson lines. We will only consider Abelian gauge groups here.

By extracting the parity-violating part of one-loop triangle diagrams with three external gauge bosons and a massive spin $1 / 2 \mathrm{KK}$ fermion of mass $m$ in the loop [14, 31], one obtains the following contribution to the low energy effective action:

$$
-\frac{\operatorname{sign} m}{2} q^{i} q^{j} q^{k} \frac{1}{6} \int A^{i} \wedge F^{j} \wedge F^{k}
$$

where the fermion couples with charge $q_{i}$ to the gauge-field $A^{i}$. When the fermion arises as a KK mode of a chiral fermion in six-dimensions, the sign of KK mass is correlated with its chirality: positive for positive and negative for negative. In the context of this discussion, the charged fermions contributing to the CS level arise only from hypermultiplets. They have negative chirality. Below, when we consider non-Abelian gauge groups and Wilson lines, we also need to include the fermions from vector multiplets. They have positive chirality. 
The CS level, defined via

$$
-k_{i j k} \frac{1}{6} \int A^{i} \wedge F^{j} \wedge F^{k}
$$

is obtained by summing over the KK spectrum. This sum diverges and needs to be regularised. We will use $\zeta$-function regularisation. We will comment on this bellow.

We now compute the CS levels $k_{i j k}$ for a collection of hypermultiplets $H_{I}, I=1, \ldots, n_{\mathrm{H}}$ with charge vectors $\vec{q}_{I}=\left(q_{I}^{1}, \ldots, q_{I}^{r}\right)$ under $\mathrm{U}(1)^{r}$. We find

$$
k_{i j k}=-2 \cdot \frac{1}{2}\left(\sum_{I} q_{I}^{i} q_{I}^{j} q_{I}^{k}\right)\left(\sum_{n=1}^{\infty} 1\right) .
$$

Here $n \in \mathbb{Z}_{+}$is the KK level. We have used that each hypermultiplet contains a pair of negative-chirality MW-fermions, hence the overall factor of 2 and $\operatorname{sign}\left(m_{n}\right)=-1$. Using $\sum_{n=1}^{\infty, \text { reg }} 1=-\frac{1}{2}$ we obtain

$$
k_{i j k}=\frac{1}{2} \sum_{I} q_{I}^{i} q_{I}^{j} q_{I}^{k}
$$

After this review, we now consider the general relation between five and six-dimensional theories in the M/F theory framework. Non-Abelian gauge groups appear due to singularities of the elliptically-fibered CY manifold on which the theory is compactified. It is known that, in general, resolving the singularities does not preserve the elliptic structure. On the M-theory side, nothing special happens, and one simply moves along the Coulomb branch, where at a generic point the non-Abelian gauge groups are broken to their maximal tori, and the theory has only U(1) factors. This theory does not seem to have a naive F-theory dual, however it can be seen as the six-dimensional $\mathcal{N}=1$ theory compactified on $S^{1}$ with Wilson lines turned on. Indeed, the motions on the five-dimensional Coulomb branch are parametrised by the scalars in $5 \mathrm{~d}$ vector multiplets. Their six-dimensional origin is as Wilson lines of $6 \mathrm{~d}$ vector multiplets, which themselves have no scalar component, when compactified on $S^{1}$. Therefore, turning on a Wilson line on the $\mathrm{F}$ theory side naturally translates into resolving singularities of the internal space on the M theory side.

The simplest yet not entirely trivial case allows to verify this understanding. Consider an $A_{1}$ singularity along a genus $g$ curve on the base of an elliptically fibered $\mathrm{CY}_{3}$. This yields a six-dimensional theory with one $\mathrm{SU}(2)$ gauge multiplet and $g$ hypermultiplets in the adjoint representation. Now we turn on a Wilson line of type $\operatorname{diag}(-\phi, 0, \phi)$, where $0<\phi<\frac{\pi}{r}$, and $r$ is the radius of the $S^{1}$. This breaks $\mathrm{SU}(2)$ to $\mathrm{U}(1)$. The resulting KK spectrum and the respective contributions to five-dimensional Chern-Simons terms comprise:

- $g$ massive hypermultiplets with $\mathrm{U}(1)$ charge $q=2$. Their KK masses are $(-\phi,-\phi-$ $\left.\frac{2 \pi}{r},-\phi-\frac{4 \pi}{r}, \ldots\right)$. They induce a Chern-Simons term with level ${ }^{18}$

$$
k_{H}^{+}=-4 g
$$

\footnotetext{
${ }^{18}$ Here we use $\sum_{n=0}^{\infty, \text { reg }} \operatorname{sign}(n+x)=\zeta(0, x)=\frac{1}{2}-x$ for $0<x<1$ and we drop the $\phi$ dependent part (contained in $x$ ). We know that the theory is anomaly free and this part will cancel via the GS mechanism. See also the discussion in section 2.2 .
} 
- $g$ massive hypermultiplets with charge $q=-2$ and the KK mass spectrum $(\phi-$ $\left.\frac{2 \pi}{r}, \phi-\frac{4 \pi}{r}, \ldots\right)$. The induced Chern-Simons term has level

$$
k_{H}^{-}=-4 g
$$

- Positive chirality fermions in the vector multiplet with $\mathrm{U}(1)$ charges \pm 2 . Their contribution to the Chern-Simons term is obtained from that of the hypermultiplets by taking into account an overall minus sign due to opposite chirality and therefore opposite sign of the KK masses and that their multiplicity is one rather than $g$.

Summing all contributions results in

$$
8(1-g)\left(-\frac{1}{6} A \wedge F \wedge F\right)
$$

This one loop calculation can be matched by a geometric one on the M theory side if we consider the Calabi-Yau manifold after resolving the $A_{1}$ singularity. Denoting the resolution divisor be $E$, one computes the coefficient of the corresponding Chern-Simons as $E \cdot E \cdot E=8(1-g)$ [33]. Moreover, it should also be clear that the geometric counterpart of changing the sign of the Wilson line $\operatorname{diag}(-\phi, 0, \phi) \rightarrow \operatorname{diag}(\phi, 0,-\phi)$, which reverses the sign of the one-loop calculation of the Chern-Simons level $8(1-g) \rightarrow 8(g-1)$, is an elementary transformation on the resolved Calabi-Yau side. This interpretation is compatible with the fact that the singular $\mathrm{CY}_{3}$ we start from should be thought of as sitting on the boundary of the Kähler cone on the M theory side.

Even though we consider compact elliptically fibered $\mathrm{CY}_{3}$, the result is essentially the same as in [33], where five-dimensional SYM was obtained from M-theory on noncompact $\mathrm{CY}_{3}$.

A final remark is in order. In above calculations we used a specific regularisation, and the values of the Chern-Simons couplings depend of this choice. The regularisation must be such that they are properly quantised. When using a different regularisation scheme, this corresponds in the dual picture to a shift of the corresponding divisor $E \rightarrow E+B$ where $B$ is a $\left(\mathbb{Q}\right.$-)divisor ${ }^{19}$ as already remarked in [13]. On the UV side (i.e. the full $\mathrm{M} / \mathrm{F}$ theory picture) we know that the KK vector corresponds to the Poincaré dual $\mathrm{PD}[B]$, while on the IR side, the result obtained by applying zeta-function regularisation, corresponds to a shifted divisor. As explained in section 3.3, the correct shift respecting the standard $\mathrm{U}(1)$ quantisation $F \in H^{2}\left(M_{5}, \mathbb{Z}\right)$ is given by $2 \mathrm{PD}[B]+c_{1}(B)$. However, on the $\mathrm{CY}_{3}$ side, $E \cdot E \cdot E=8(1-g)$ appears to be 'rigid'.

\subsection{Unitarity condition for linear BPS strings}

Before considering five-dimensional theories in detail, we recall the unitarity condition for $6 \mathrm{~d} \mathcal{N}=1$ supergravity theories proposed in [18]. The anomaly polynomial for the worldsheet theory, which can be computed from anomaly inflow from the bulk, was already given

\footnotetext{
${ }^{19}$ Special attention needs to be paid to the quantisation condition related to the corresponding magnetic charge.
} 
in eq. (3.4). From this we need to subtract the contribution of a free $(0,4)$ hypermultiplet, whose bosonic components describe the position of the string in the four transverse directions:

$$
I_{4}^{\text {free }}=-\frac{1}{12} p_{1}\left(T W_{2}\right)-c_{2}(1) .
$$

Recall that $c_{2}(1)$ and $c_{2}(2)$ correspond to the subbundles of the normal bundle $\mathrm{SO}(4) \cong$ $\mathrm{SU}(2)_{1} \times \mathrm{SU}(2)_{2}$. We identify $\mathrm{SU}(2)_{1}$ with the $\mathrm{SU}(2)_{\mathcal{R}}$-symmetry of the interacting $(0,4)$ SCFT in the IR. The anomaly polynomial of the interacting theory is then (cf. also section 3.1 for further details on the notation)

$$
\begin{aligned}
I_{4}^{\mathrm{int}}= & -\frac{1}{12}(3 Q \cdot a-1) p_{1}\left(T W_{2}\right)+\sum_{i} Q \cdot b_{i} \frac{1}{4 h_{i}^{\vee}} \operatorname{Tr}_{\text {Adj }}\left(F_{G_{i}}^{2}\right) \\
& -\frac{1}{2}(Q \cdot Q-Q \cdot a) c_{2}(1)+\frac{1}{2}(Q \cdot Q+Q \cdot a+2) c_{2}(2) \\
\supset & -\frac{1}{24}\left(c_{R}^{\mathrm{int}}-c_{L}^{\mathrm{int}}\right) p_{1}\left(T W_{2}\right)+\sum_{i} k_{i} \frac{1}{4 h_{i}^{\vee}} \operatorname{Tr}_{\mathrm{Adj}}\left(F_{G_{i}}^{2}\right)-k_{\mathcal{R}} c_{2}\left(\mathrm{SU}(2)_{\mathcal{R}}\right) .
\end{aligned}
$$

Note that the positivity of the central charge of the $\mathrm{SU}(2)_{2}$ current algebra requires $Q \cdot Q+Q \cdot a+2 \geq 0$.

This leads to the expression for the central charges of the interacting SCFT

$$
\begin{gathered}
c_{L}^{\mathrm{int}}-c_{R}^{\mathrm{int}}=-6 Q \cdot a+2 \\
c_{R}^{\mathrm{int}}=6 k_{\mathcal{R}}=3(Q \cdot Q-Q \cdot a)
\end{gathered}
$$

and to the unitarity requirement

$$
\sum_{i} \frac{\left(Q \cdot b_{i}\right) \cdot \operatorname{dim} G_{i}}{Q \cdot b_{i}+h_{i}^{\vee}} \leq c_{L}^{\mathrm{int}}=3 Q \cdot Q-9 Q \cdot a+2
$$

where we have used the relation between the levels $k_{i}=Q \cdot b_{i}$ of the left-moving current algebras and their contribution to the central charge. In general, eq. (4.11) gives strong constraints when the charge $Q$ is small.

As discussed in section 3.1, when putting this $6 \mathrm{~d}$ supergravity on a circle transverse to the string, we identify $c_{2}(1)=c_{2}(2)=c_{2}\left(\mathrm{SU}(2)_{\mathcal{R}}\right)$. The resulting central charges are then:

$$
c_{R}=6 k_{R}=-6 Q \cdot a \quad \text { and } \quad c_{L}=-12 Q \cdot a .
$$

Again, subtracting the free part of the central charge, we have

$$
c_{R}^{\mathrm{int}}=-6 Q \cdot a-6, \quad c_{L}^{\mathrm{int}}=-12 Q \cdot a-3 .
$$

On the other hand, the gauge anomaly should not be changed by compactifying our theory on a circle. Since if a $6 \mathrm{~d}$ theory is good, it should also be good after $S^{1}$ compactification, so we derive the following unitarity condition:

$$
\sum_{i} \frac{\left(Q \cdot b_{i}\right) \cdot \operatorname{dim} G_{i}}{Q \cdot b_{i}+h_{i}^{\vee}} \leq c_{L}^{\mathrm{int}}=-12 Q \cdot a-3 .
$$


A remark is in order here. Notice that the above central charge calculation in $5 \mathrm{~d}$ differs from the $6 \mathrm{~d}$ case. First, in $6 \mathrm{~d}$ there is a second $\mathrm{SU}(2)$ on the right moving side while in 5 d generically we only have $\mathrm{SU}(2)_{\mathcal{R}}$ symmetry. Second, the -3 contribution which appears in $5 \mathrm{~d}$ central charge $c_{L}^{\text {int }}$ is due to the fact that for the $5 \mathrm{~d}$ strings we only have three transverse bosons on the left moving side. The left moving compact boson from compact transverse circle may belong to the interacting part of the CFT. However, due to the $(0,4)$ supersymmetry, the right moving compact boson should sit in the free hypermultiplet together with the other three right moving transverse bosons.

\subsection{Charges of supergravity strings}

In order to use the unitarity condition (4.13), we must find a way to single out supergravity strings [19] (i.e. strings that cannot be consistently decoupled from gravity). In order to read off the central charge of the $(0,4)$ SCFT on the BPS string, it is essential to indentify the $\mathrm{SU}(2)_{\mathcal{R}}$ symmetry of the $(0,4) 2 \mathrm{~d}$ SCFT with the structure (sub)group from the normal bundle. However, as [18, 19] already pointed out that for the BPS strings that can be consistently decoupled from gravity (i.e. BPS strings in $6 \mathrm{~d} / 5 \mathrm{~d}$ SCFT ), the $\mathrm{SU}(2)_{\mathcal{R}}$ symmetry of the $(0,4) 2 \mathrm{~d}$ SCFT may no longer come from the structure (sub)group of the normal bundle (for example, it may be mixed with the SU(2) R-symmetry from the bulk in the SCFT limit).

The conditions for the six-dimensional $\mathcal{N}=1$ theory to have a well defined moduli space were analysed in [18], and can be summarise using a $\left(1, n_{T}\right)$ vector $j$ (related to the Kähler form on the base of the elliptic fibration $B$ ) on the tensor branch of the theory

$$
j \cdot j>0, \quad j \cdot b_{i}>0, \quad j \cdot a<0 .
$$

In order for the string to have a non-negative tension, $j \cdot Q \geq 0$ also needs to be imposed. Finally, unitarity of the $(0,4) 2 \mathrm{~d}$ SCFT hosted on the BPS string imposed

$$
Q \cdot Q+Q \cdot a \geq-2, \quad Q \cdot a<0 \text { and } Q \cdot b_{i} \geq 0
$$

as $k_{i}=Q \cdot b_{i}$ is the level of affine current algebra on the left moving side of the $(0,4)$ SCFT and $c_{R}=-6 Q \cdot a$ for the $(0,4) 5 \mathrm{~d}$ BPS strings that comes from $(0,4)$ BPS strings in $6 \mathrm{~d}$ after circle compactification. Any five-dimensional theory obtained from a circle reduction should also be subject to these constraints. As our main interest is in BPS strings in $6 \mathrm{~d} \mathcal{N}=1$ supergravity and their counterpart in $5 \mathrm{~d} \mathcal{N}=1$ supergravity after circle compactification, we shall impose the above conditions (4.14) and (4.15).

Now we shall argue that non-negative $Q \cdot Q$ is a sufficient condition for a BPS string to be identified as a supergravity string. This argument is carried out in two steps:

- First, notice that the strings which can be decoupled from gravity (i.e. not supergravity strings) must go tensionless at some point of the Kähler moduli space.

- Then we argue the strings with $Q \cdot Q \geq 0$ can never go tensionless on the Kähler moduli space at any finite distance point. 
As a byproduct of this discussion, we can show that all the $5 \mathrm{~d} U(1)$ gauge fields from $6 \mathrm{~d}$ tensors associated with supergravity strings, can never be enhanced to non-Abelian gauge fields in 5d supergravity. When gravity is decoupled, the $\mathrm{U}(1)$ 's related to the supergravity strings will also decouple. The U(1)'s sourced by the other strings may be enhanced to non-Abelian gauge fields in the field theory limit.

Which strings can be consistently decoupled from gravity? The energy scale associated to a magnetic string is given by its tension $T$, while gravity sets the energy scale $M_{\mathrm{Pl}}$. In order for a string to decouple from gravity, it should be possible to take the limit $\frac{T}{M_{\mathrm{Pl}}} \rightarrow 0$, where the backreaction of the string can be neglected. Working in the supergravity regime, we may chose to keep $M_{\mathrm{Pl}}$ fixed and, as a result, need to have $T \rightarrow 0$ in the decouplings limit. One may equivalently state:

Any string that can be decoupled from gravity, must go tensionless at some point of the Kähler moduli space.

For six-dimensional theories obtained from F-theory on a Kähler base $B$ of a elliptically fibered Calabi-Yau manifold, this can be also understood geometrically. The string source is given by a D3-brane wrapping a curve $D \subseteq B$, and the two energy scales

$$
T \sim \operatorname{vol}(D), \quad M_{\mathrm{P} 1} \sim \operatorname{vol}(B)
$$

Usually in order to go to the field theory (decoupling) limit, one takes $\operatorname{vol}(B) \rightarrow \infty$, i.e. the internal manifold is taken to be non-compact. Here instead we take $\operatorname{vol}(B)=1$ (which is $j \cdot j=1$ for $j$ a $\left(1, n_{T}\right)$ vector which parameterizes the Kähler moduli space in $6 \mathrm{~d} \mathcal{N}=1$ supergravity language). Then the decoupling is achieved by

$$
\operatorname{vol}(D) \rightarrow 0,
$$

which indicates that the submanifold $D$ on which D3 wraps should be shrinkable. This is equivalent to $D \cdot D<0$ and translates into the condition $Q \cdot Q<0$ for the BPS string charge $Q$. Such strings should be excluded from our analysis.

On the contrary, when a D3 brane wraps a semi-ample divisor, we expect to have a supergravity string [19] that cannot be decoupled from gravity consistently and is subject to the unitarity constraints. An semi-ample divisor is not shrinkable while keeping the base being an algebraic surface, and has the property $D \cdot D \geq 0 \Longleftrightarrow Q \cdot Q \geq 0$. We also assume the divisor which the D3 brane wraps is irreducible.

Strings with $Q \cdot Q \geq 0$ are supergravity strings. In order to see that strings with $Q \cdot Q \geq 0$ will not become tensionless on the Kähler moduli space, first recall that the string tension is given by $j \cdot Q$. We have fixed $j \cdot j=1$, and can now choose the inner product and Kähler parameter to be respectively:

$$
\Omega=\operatorname{diag}(1,-1, \ldots,-1), \quad \text { and } \quad j=\left(\sqrt{|\vec{j}|^{2}+1}, \vec{j}\right) .
$$

If $Q \cdot Q \geq 0$, we may take:

$$
Q=\left(\sqrt{|\vec{Q}|^{2}+m}, \vec{Q}\right)
$$

where $m$ is a non-negative integer. 
Now the tension can be evaluated directly:

$$
j \cdot Q=\sqrt{|\vec{j}|^{2}+1} \cdot \sqrt{|\vec{Q}|^{2}+m}-\vec{Q} \cdot \vec{j} \geq \sqrt{|\vec{j}|^{2}+1} \cdot \sqrt{|\vec{Q}|^{2}+m}-|\vec{j}| \cdot|\vec{Q}|>0
$$

and is strictly positive on the Kähler moduli space. One may, of course, have $|\vec{j}| \rightarrow$ $\infty$ at infinite distance at the boundary of the moduli space. However, there the entire effective supergravity description may break down and the full stringy picture needs to be considered, very much in analogy with the distance conjecture. As a result, in the supergravity theory, that we are considering, these BPS strings cannot go to tensionless limit and cannot be consistently decoupled from gravity. An alternative proof of this is given in appendix B.

We close this section with two remarks. The first concerns the comparison of the condition of non-negativity of $Q \cdot Q$ that we imposed with the conditions which appeared in the analysis of [18], The second addresses the possibility of symmetry enhancement in fivedimensional theories obtained from a circle reduction of $(0,1)$ theories in six dimensions.

Strings with $\boldsymbol{Q} \cdot \boldsymbol{Q}=\mathbf{- 1}$. It was pointed out in [18] that the positivity of the rightmoving central charge and the positivity of the central charge associate to the $\mathrm{SU}(2)_{2}$ current algebra yield $Q \cdot Q-Q \cdot a \geq 0$, and $Q \cdot Q+Q \cdot a \geq-2$ respectively. This leads to the necessary condition $Q \cdot Q \geq-1$ for the unitarity of the worldsheet SCFT.

We have already seen above that $Q \cdot Q \geq 0$ corresponds to supergravity strings, and may now consider BPS strings with string charge $Q \cdot Q=-1$. The unitarity condition is not applicable since such strings may be consistently decoupled form gravity, As a result, the $\mathrm{SU}(2)_{R}$ symmetry of the related $(0,4)$ SCFT may not be identifiable with the $\mathrm{SU}(2)$ from normal bundle, as it was already pointed out in [18].

As our central charge formula holds for supergravity strings and may not be applied to BPS strings that can be consistently decoupled from gravity, we will focus on BPS strings with charge $Q \cdot Q \geq 0$. We will also see in the next section that this property has a clear counterpart on the elliptic $\mathrm{CY}_{3}$ for the F-theory model. It translates into the requirement that the divisor $D$ on the base of the elliptically-fibered $\mathrm{CY}_{3}$, which is wrapped by a D3-brane, is nef (or semi-ample). We can also see why strings with charges satisfying $Q \cdot Q=-1$ may be consistently decoupled from gravity in F-theory models: in this case the corresponding divisors that D3 branes wrap are shrinkable on the base.

Supergravity strings and gauge symmetry (non)enhancement after circle reduction. When the six-dimensional $(0,1)$ theory is put on a circle, the resulting fivedimensional supergravity theory has a memory of the original $\mathrm{SO}\left(1, n_{T}\right)$ symmetry [6]. But other than that, the reduction of each self-dual tensor field yields a $\mathrm{U}(1)$ gauge field that is not very different from other vector fields. Hence one may wonder if there are special points in the moduli space where this $\mathrm{U}(1)^{n_{T}}$ symmetry maybe enhanced.

Let us assume that such enhancement is possible, and that one can have a theory with gauge group $G$. If so, moving on the Coulomb branch of the $5 \mathrm{~d}$ theory away from the special locus, the gauge group will break to its maximal torus $G \rightarrow \mathrm{U}(1)^{\mathrm{rk}(G)}$. This process will produce electric BPS particles carrying charges $\pm Q$ which belong to the root 
lattice of the gauge group $G$. The simplest example of this type is $\mathcal{N}=1 \mathrm{SU}(2)$ gauge theory, where the corresponding electric BPS particles are W-bosons with charges \pm 2

On the other hand, if any of these U(1)s originate from $6 \mathrm{~d}$ tensors, we should be able to identify these $5 \mathrm{~d}$ electric BPS particles with charge $\pm Q$ as $6 \mathrm{~d}$ BPS strings with charge $\pm Q$ wrapping the circle. So in the six-dimensional spectrum, BPS strings with both $Q$ and $-Q$ should appear. As mentioned, the non-negativity of the string tension requires $j \cdot Q \geq 0$. And the only consistent way to reconcile these conditions is to have $j \cdot Q=0$ at some points of the Kähler moduli space, which means these strings can be consistently decoupled from gravity. So such enhancement is only possible in the field theory limit.

Since the U(1) gauge fields, under which the supergravity strings are charged, cannot be decoupled from gravity, any symmetry enhancement involving these will not be compatible with the six-dimensional BPS spectrum. Thus, these U(1)'s cannot be enhanced to nonAbelian gauge groups in the supergravity regime.

As we shall see, due to the absence of the quadratic piece $Q \cdot Q$ in the unitarity condition, the five-dimensional unitarity condition is in general stronger than the six-dimensional condition of [18]. The only exception to this is when $Q \cdot Q+Q \cdot a=-2$. When this holds, the $6 \mathrm{~d}$ unitarity condition imposes slightly stronger constraints than the $5 \mathrm{~d}$ one.

\section{Unitarity condition as a weak Kodaira positivity condition}

In the F-theory framework, the upper bound on the rank and the type of non-Abelian gauge groups in six-dimensional $\mathcal{N}=1$ theories arises naturally, and is due to the purely geometric condition, the Kodaira positivity (KPC), on the elliptically fibered threefold. The purpose of this section is to compare the implications of the KPC with the unitarity condition (UC) discussed in section 4. This comparison will be complete for the theories without Abelian gauge groups. This does not lead to a significant loss of generality due to the fact that generally $\mathrm{U}(1)$ factors in F-theory models appear due to Higgsing of a non-Abelian gauge group as argued in $[36] .^{20}$

In order to carry out this comparison we should rewrite the UC (4.13)

$$
\sum_{i} \frac{\left(Q \cdot b_{i}\right) \cdot \operatorname{dim} G_{i}}{Q \cdot b_{i}+h_{i}^{\vee}} \leq-12 Q \cdot a-3
$$

in a more convenient form. This is possible due to the fact that it involves $-12 Q \cdot a$. In the F-theoretic models, when mapping the anomaly data to the geometric data of ellipticallyfibered $\mathrm{CY}_{3}, a$ is mapped to the canonical divisor $K$. The fact, that the elliptic fibration requires that all the gauge divisors should be contained in the effective divisor $-12 \mathrm{~K}$, hints at a possible interpretation of the UC as a physical counterpart of the purely geometrical KPC. If the six-dimensional minimally-supersymmetric theory is obtained from F-theory on an elliptically-fibered $\mathrm{CY}_{3}$, the comparison is direct (as we shall see in section 5.2). However, the UC should apply without any assumption on the model having a F-theory realisation.

\footnotetext{
${ }^{20}$ The bounds on the number of $\mathrm{U}(1)$ factors were studied in $[34,35]$.
} 
A remark on notation: in F-theoretic models, we are interested in the BPS strings that originate from D3-branes wrapping effective divisors in the base manifold $B$. As we shall see these BPS objects correspond to supergravity strings when the divisor in question is semi-ample, i.e. the linear system associated to a positive power of this divisor is base-point free. On the other hand, all effective nef divisors, i.e. the divisors that have a nonnegative intersection with every curve in $B$, are semi-ample. Since we are discussing only the effective divisors wrapped by D3-branes we just use the label nef divisors, hopefully without causing any confusion.

\subsection{Rewriting the unitarity condition}

The Kodaira positivity condition states that all singular divisors should be contained in the divisor of the discriminant of the Weierstrass model. ${ }^{21}$ This requires the residual divisor $Y$ given by

$$
Y=-12 K-\sum_{i} x_{i} S_{i}
$$

to be effective.

Here $S_{i}$ are the divisors with singular elliptic curve that host non-Abelian gauge groups [37] and $x_{i}$ is the vanishing order of the discriminant on $S_{i}($ i.e. $\operatorname{ord}(\Delta)$ in table 1). Every effective divisor satisfies

$$
j_{B} \cdot\left(-12 K-\sum_{i} x_{i} S_{i}\right)=j_{B} \cdot Y \geq 0,
$$

where $j_{B}$ is the Kähler form on the base $B$. In fact, the following

$$
D \cdot\left(-12 K-\sum_{i} x_{i} S_{i}\right)=D \cdot Y \geq 0
$$

holds for any nef (or semi-ample) divisor $D$, as nef divisor should intersect every effective divisor non-negatively.

One can recast this condition in a form that just uses the data of six-dimensional supergravity, notably the four-form $X_{4}^{\alpha}$ entering the anomaly polynomial, and does not invoke the elliptically fibered $\mathrm{CY}_{3}$ explicitly [8]

$$
j \cdot\left(-12 a-\sum_{i} x_{i} S_{i}\right) \geq 0
$$

\footnotetext{
${ }^{21} \mathrm{We}$ are using the standard conventions for the elliptic fibrations with section (see e.g. [10, 11]). The elliptical fiber on a $\mathrm{CY}_{3}$ is defined by an equation

$$
y^{2}=x^{3}+f(u, v) x+g(u, v)
$$

in an affine patch of the weighted projective space $\mathbb{W P}_{2,3,1}$, with $u$ and $v$, one set of affine coordinates on the base $B$, fixed. Note, $f \in \Gamma(-4 K)$ and $g \in \Gamma(-6 K)$. The degeneration loci of the elliptic fiber are given by zeros of the discriminant:

$$
\Delta=4 f^{3}(u, v)+27 g^{2}(u, v),
$$

and, $\Delta \in \Gamma(-12 K)$.
} 
where $j$ is a $\left(1, n_{T}\right)$ vector on the tensor branch of our six-dimensional theory which satisfies $j \cdot j>0, j \cdot b_{i} \geq 0, j \cdot a<0$. For any $6 \mathrm{~d}$ minimal supergravity theory not obtained from an elliptically-fibered $\mathrm{CY}_{3}$, condition (5.5) would appear to be not physically motivated and does not have to be satisfied.

On the other hand, the condition (5.1) follows from the worldsheet unitarity of supergravity strings and is expected to hold for all $6 \mathrm{~d}$ minimal supergravity theories that are consistent at quantum level. When applied to an F-theoretic model, it can be rewritten as

$$
D \cdot\left(-12 K-\sum_{i} S_{i} \frac{\operatorname{dim} G_{i}}{D \cdot S_{i}+h_{i}^{\vee}}\right) \geq 3
$$

One has to bear in mind that the divisor $D$ is wrapped by a D3-brane, and is required to be nef as we are talking about supergravity strings. To see why this is so, recall that the direct analogue of $Q \cdot Q \geq 0$ for the supergravity charges is given by $D \cdot D \geq 0$ for an irreducible effective divisor $D$. Irreducibility of $D$ will be assumed throughout this paper.

Without assuming that the $6 \mathrm{~d}$ minimal supergravity theory has an F-theoretic origin, one still needs to augment (5.1) by the following:

$$
Q \cdot Q+Q \cdot a+2 \geq 0, \quad k_{i}=Q \cdot b_{i} \geq 0 \quad \text { and } \quad-Q \cdot a>0
$$

These can be interpreted as constraints on admissible values of the charge $Q$, in addition to $Q \cdot Q \geq 0$. The first two conditions are the requirements that the levels of current algebras are larger than 0 , while the last one is the positivity of the right-moving central charge of the $(0,4)$ worldsheet theory (recalling $c_{R}=-6 Q \cdot a$ after the circle compactification).

It is not hard to see that the strongest constraints following from (5.1) are when $Q \cdot b_{i}=1$ in the denominator (although $Q \cdot b_{i}=1$ may not be achieved as intersection of divisors of a base $B$ when we consider $\mathrm{F}$ theory model). In the following we shall compare the KPC with the following

$$
Q \cdot\left(-12 a-\sum_{i} b_{i}\left(\frac{\operatorname{dim} G_{i}}{1+h_{i}^{\vee}}\right)\right) \geq 3 .
$$

Indeed when this condition is satisfied, (5.1) will hold also for $Q \cdot b_{i}>1$.

The failure of the (5.8) to hold does not immediately signify any inconsistency. Indeed, one has to first verify that $Q \cdot b_{i}=1$ is possible. ${ }^{22}$ We shall see that in general (5.8) is weaker than $\mathrm{KPC}$, and hence it may serve as a useful measure for the $6 \mathrm{~d}$ minimal supergravity theories that have no F-theoretic realisation. On the contrary, for the $6 \mathrm{~d}$ theories originating from $\mathrm{F}$ theory, (5.8) may provide finer information about the effective divisor $Y=-12 K-\sum_{i} x_{i} S_{i}$ in some special cases, where the constraints imposed by the $\mathrm{UC}$ turn out to be stronger than those following from KPC.

\footnotetext{
${ }^{22}$ We will do the full comparison between $\mathrm{UC}$ (rather than its strongest version as here) and KPC in 5.2.2. So the UC in the strong form (5.8) serves as a red flag: if the strong condition fails, UC as given in (5.1) should be checked. In fact we have found situations where it fails, but $Q \cdot b_{i}=1$ fails as well.
} 


\begin{tabular}{|c|c|c|c|c|c|c|c|c|}
\hline type & $\operatorname{ord}(f)$ & $\operatorname{ord}(g)$ & $\operatorname{ord}(\Delta)$ & sing. & $\mathfrak{g}$ & split & $y=\frac{\operatorname{dim} G}{1+h^{V}}$ & $K$-type \\
\hline$I_{0}$ & $\geq 0$ & $\geq 0$ & 0 & - & - & & - & - \\
\hline$I_{1}$ & 0 & 0 & 1 & - & - & & - & - \\
\hline$I I$ & $\geq 1$ & 1 & 2 & - & - & & - & - \\
\hline$I I I$ & 1 & $\geq 2$ & 3 & $A_{1}$ & $s u(2)$ & & 1 & $K_{2}$ \\
\hline$I V$ & $\geq 2$ & 2 & 4 & $A_{2}$ & $\begin{array}{l}s p(1) \\
s u(3)\end{array}$ & $\begin{array}{l}I V^{n s} \\
I V^{s}\end{array}$ & $\begin{array}{l}1 \\
2\end{array}$ & $K_{2}$ \\
\hline$I_{m}$ & 0 & 0 & $m$ & $A_{m}$ & $\begin{array}{l}s p\left(\left[\frac{m}{2}\right]\right) \\
s u(m)\end{array}$ & $\begin{array}{l}I_{m}^{n s} \\
I_{m}^{s} \\
\end{array}$ & $\begin{array}{c}2\left[\frac{m}{2}\right]-\frac{3\left[\frac{m}{2}\right]}{\left[\frac{m}{2}\right]+2} \\
m-1\end{array}$ & $\begin{array}{c}K_{1} / K_{2} \\
K_{1}\end{array}$ \\
\hline$I_{0}^{*}$ & $\geq 2$ & $\geq 3$ & 6 & $D_{4}$ & $\begin{array}{l}g_{2} \\
s o(7) \\
s o(8)\end{array}$ & $\begin{array}{l}I_{0}^{* n s} \\
I_{0}^{* s s} \\
I_{0}^{* s}\end{array}$ & $\begin{array}{c}14 / 5 \\
7 / 2 \\
4\end{array}$ & $K_{2}$ \\
\hline $\begin{array}{l}I_{2 n-5}^{*}, \\
n \geq 3\end{array}$ & 2 & 3 & $2 n+1$ & $D_{2 n-1}$ & $\begin{array}{l}s o(4 n-3) \\
s o(4 n-2)\end{array}$ & $\begin{array}{l}I_{2 n-5}^{* n s} \\
I_{2 n-5}^{* s} \\
\end{array}$ & $\begin{array}{c}2 n-3 / 2 \\
2 n-1\end{array}$ & $K_{2}$ \\
\hline $\begin{array}{l}I_{2 n-4}^{*}, \\
n \geq 3\end{array}$ & 2 & 3 & $2 n+2$ & $D_{2 n}$ & $\begin{array}{l}s o(4 n-1) \\
s o(4 n)\end{array}$ & $\begin{array}{l}I_{2 n-4}^{* n s} \\
I_{2 n-4}^{* s}\end{array}$ & $\begin{array}{c}2 n-1 / 2 \\
2 n\end{array}$ & $K_{2}$ \\
\hline$I V^{*}$ & $\geq 3$ & 4 & 8 & $E_{6}$ & $\begin{array}{l}f_{4} \\
e_{6}\end{array}$ & $\begin{array}{l}I V^{* n s} \\
I V^{* s}\end{array}$ & $\begin{array}{c}52 / 10 \\
6\end{array}$ & $K_{2}$ \\
\hline$I I I^{*}$ & 3 & $\geq 5$ & 9 & $E_{7}$ & $e_{7}$ & & 7 & $K_{2}$ \\
\hline$I I^{*}$ & $\geq 4$ & 5 & 10 & $E_{8}$ & $e_{8}$ & & 8 & $K_{2}$ \\
\hline non-min. & $\geq 4$ & $\geq 6$ & $\geq 12$ & non-can. & - & & - & - \\
\hline
\end{tabular}

Table 1. The left side of this table summarises the Kodaira-Tate data for singular fibers of the Weierstrass model. The Weierstrass data $f, g$ and $\Delta$ define the type of singularity. Some of the singularities can lead to different gauge algebras. This is governed by the refined Tate fiber type (see e.g. [11] for details). In the last column of the left side of the table, $n s, s$ and ss stand for non-split, split and semi-split respectively. In our context the most important column is $\operatorname{ord}(\Delta)$ which defines the $x_{i}$ multiplicities of the divisors with singular fibers $S_{i}$. The right side of the table summarises the values of $y_{i}$ multiplicities that appear in the UC. The last column, $K$-type, is determined by the difference $x_{i}-y_{i}$ (see also table 2).

\subsection{Comparing KPC and UC}

It is useful to recall the types of singularities present in the elliptically fibered $\mathrm{CY}_{3}$ and the ensuing local gauge groups. These are conveniently summarised by the Kodaira data and can be found in table 1, which we have augmented by some data entering the UC.

We can directly compare the quantity $y_{i}=\frac{\operatorname{dim} G^{i}}{1+h_{i}^{\vee}}$ and the Kodaira multiplicity $x_{i}=$ $\operatorname{ord}(\Delta)$ one by one:

- For $E_{6,7,8}$, we have $y_{E_{6,7,8}}=x_{E_{6,7,8}}-2=6,7,8$;

- For $\mathrm{SU}(n \geq 2)$, we have $y_{\mathrm{SU}(n)}=n-1=x_{\mathrm{SU}(n)}-1$ for type $I_{n}$ and $y_{\mathrm{SU}(2)}=1=$ $x_{\mathrm{SU}(2)}-2$ for type $I I I, I V$; 


\begin{tabular}{|c|c|c|}
\hline Type of gauge algebra & $x_{i}-y_{i}$ & Gauge algebra \\
\hline$K_{1}$ & $<2$ & $s u(m), s p(1), s p(2), s p(3)$ in Kodaira type $I$ \\
\hline$K_{2}$ & $\geq 2$ & All other groups in table 1 \\
\hline
\end{tabular}

Table 2. Classification of gauge groups in table 1 based on the minimal value of $x_{i}-y_{i}$. Notice that $s p(1), s p(2), s p(3)$ in the first row come from $I_{2}, I_{4}, I_{6}$ respectively.

- For $F_{4}, G_{2}$, we have $y_{F_{4}, G_{2}}=\frac{14}{5}, \frac{52}{10}$ while $x_{F_{4}, G_{2}}=6,8$;

- For $\mathrm{SO}(2 n+1), n \geq 3$, we have $y_{\mathrm{SO}(2 n+1)}=n+\frac{1}{2}$, while $x_{\mathrm{SO}(2 n+1)}=n+3$;

- For $\mathrm{SO}(2 n), n \geq 4$, we have $y_{\mathrm{SO}(2 n)}=n$, while $x_{\mathrm{SO}(2 n)}=n+2$;

- For $\operatorname{Sp}(k)$, we have $y_{\mathrm{Sp}(k)}=2 k-\frac{3 k}{k+2}, x_{\mathrm{Sp}(k)}=2 k, 2 k+1$.

From above, we see $x_{i}>y_{i}=\frac{\operatorname{dim} G^{i}}{1+h_{i}^{V}}$, hence we naturally have on any elliptic $\mathrm{CY}_{3}$ :

$$
D \cdot\left(-12 K-\sum_{i} y_{i} S_{i}\right)>D \cdot\left(-12 K-\sum_{i} x_{i} S_{i}\right)
$$

for any nef divisor $D$.

Given the respective forms of our unitarity condition

$$
D \cdot\left(-12 K-\sum_{i} y_{i} S_{i}\right) \geq 3
$$

and the Kodaira positivity condition:

$$
D \cdot\left(-12 K-\sum_{i} x_{i} S_{i}\right)=D \cdot Y \geq 0
$$

few more steps are needed to see which one leads to stronger constraints. The Kodaira positivity is a necessary condition that is satisfied in all elliptically fibered $\mathrm{CY}_{3}$. In cases where our unitarity constraints turns out to be weaker, we are not learning much new in the context of elliptically fibered $\mathrm{CY}_{3} \cdot{ }^{23}$ When they are stronger, it should follow that the $\mathrm{CY}_{3}$ in question should satisfy extra hidden conditions.

To proceed, notice that when the gauge algebra is $s u(m)$, as well $s p(1), s p(2), s p(3)$ when these are in Kodaira type $I$ (as opposed to $s p(1)$ in Kodaira type $I V$, su(2) in type $I I I$ and $s u(3)$ in type $I V)$, we have $y_{i}+1 \leq x_{i}<y_{i}+2$. We label gauge groups of this type as $K_{1}$. For all other gauge groups we have $x_{i} \geq y_{i}+2$, and we label these as type $K_{2}$. In the subsequent analysis we shall label the gauge group as $G=\left\{K_{1}, K_{2}\right\}$ when it can be of any type, either $K_{1}$ or $K_{2}$ (see table 2 ).

The UC applies to any supergravity theory, but the comparison to KPC requires to adapt it to the elliptically fibered $\mathrm{CY}_{3}$, where it can be formulated as a condition on divisor $D$ in the base $B$, wrapped by a D3-brane. $B$ is a smooth algebraic surface. In addition to

\footnotetext{
${ }^{23}$ These cases are important however for understanding the part of the not-swamped landscape of theories not covered by F-theory constructions.
} 
an irreducible effective divisor $D$ it has the gauge divisors $S_{i}$. The gauge divisor $S_{i}$ should also be an effective divisor so that it can be wrapped by $D 7$ branes.

We may recall that the charges for the supergravity strings $Q$ should satisfy $j \cdot Q>0$, $Q \cdot b_{i} \geq 0$ and $Q \cdot a<0$. We shall also impose $Q \cdot Q \geq 0$ (and comment on $Q \cdot Q=-1$ case momentarily). These conditions can be translated into geometric statements for the $D$

$$
D \cdot D \geq 0, \quad D \cdot S_{i} \geq 0, \quad D \cdot K<0 .
$$

These conditions already contain a great deal of information: $D$ is a nef divisor, and hence it intersects any effective divisor $E$ on the base non-negatively $D \cdot E \geq 0 .{ }^{24}$

$Q \cdot Q=-1$ case: notice that we have restricted $Q$ so that $Q \cdot Q \geq 0$. Before turning to the analysis of the conditions on $D$, we comment on $Q \cdot Q=-1$ case. For this case, we have $D \cdot D+D \cdot K=-2$ as $D \cdot D=-1$ and $D \cdot K<0$, and hence $D$ is a rational curve with self intersection -1 . So it corresponds to blowing up a point on a smooth base $\sigma: B \rightarrow B^{\prime}$, which means that this exceptional divisor can be smoothly shrunk to zero size. As a result, the corresponding string could be tensionless and be consistently decoupled from gravity (actually the metric on $B$ which gives zero size for this exceptional divisor can be interpreted as a metric on $B^{\prime}$. See $[38,39]$ and also [40] for a related physical discussion).

\subsubsection{Ample divisor $D$}

We shall start with the simplest case when D3 wraps an ample divisor $D$ in the base manifold $B$. These divisors have nice numerical properties given by:

$$
D \cdot S_{i} \geq 1, \quad D \cdot K \leq-1, \quad D \cdot D \geq 1 .
$$

thanks to the Nakai-Moishezon ampleness condition.

Using these, the Kodaira positivity condition (KPC) and the unitarity condition (UC) yield the following:

$$
\begin{aligned}
& D \cdot\left(-12 K-\sum_{i} x_{i} S_{i}\right)=D \cdot Y \geq 0 \\
& D \cdot\left(-12 K-\sum_{i} x_{i} S_{i}\right) \geq 3-\sum_{i}\left(x_{i}-y_{i}\right) D \cdot S_{i}
\end{aligned}
$$

and hence the following (in)equality

$$
3-\sum_{i}\left(x_{i}-y_{i}\right) D \cdot S_{i} \leq D \cdot Y
$$

should hold.

There are three distinct cases:

- Case 1: the entire gauge group is given by a product of three or more groups, $G_{1} \times$ $G_{2} \times \ldots \times G_{k}$ with $k \geq 3$, or is a product of two factors, one of which is necessarily of $K_{2}$ type, $G \times K_{2}$. Since $D \cdot S_{i} \geq 1$ and $x_{i}-y_{i} \geq 1$ in general (and $x_{i}-y_{i} \geq 2$ for a $K_{2}$-type group), $3-\sum_{i}\left(x_{i}-y_{i}\right) D \cdot S_{i} \leq 0$. Given $D \cdot Y \geq 0$, the condition (5.15) is automatically satisfied. In this case, KPC imposes stronger constraints than UC.

\footnotetext{
${ }^{24}$ Note that the condition $Q \cdot Q+Q \cdot a+2 \geq 0$ is automatically satisfied in F-theory models due to the adjunction formula.
} 
- Case 2: the entire gauge group is of type $K_{1} \times K_{1}$ :

- When the groups are $\operatorname{Sp}(2) \times \mathrm{Sp}(2), \operatorname{Sp}(2) \times \mathrm{Sp}(3)$ and $\mathrm{Sp}(3) \times \mathrm{Sp}(3)$, UC is not stronger than KPC as (5.15) is trivially satisfied.

- For other $K_{1} \times K_{1}$ groups, when $D \cdot S_{1}=D \cdot S_{2}=1$ (5.15) imposes $D \cdot Y \geq 1$.

- Case 3: the entire gauge group is a single factor $G$ :

- For $G \in K_{2}$ with $2 \leq x-y<3$ and $D \cdot S=1$ (5.15) imposes $D \cdot Y \geq 1$.

- For $G \in K_{1}$, when $D \cdot S=1$ (5.15) requires $D \cdot Y \geq 2$.

- For $\mathrm{Sp}(1)$ and $\mathrm{SU}(m)$ only, when $D \cdot S=2$, (5.15) requires $D \cdot Y \geq 1$.

As we see, Case 2 and Case 3 may contain examples where $D \cdot Y$ is strictly positive, as opposed to being simply non-negative as required by KPC. These would be at the center of our attention. Notice that in all these cases a small value of $D \cdot S_{i}$ is required. When $D \cdot S_{i}=1$, the strong version of UC (5.8) and the general UC (5.1) coincide. $D \cdot S_{i}=2$, which applies only to $G=\mathrm{Sp}(1)$ and $G=\mathrm{SU}(m)$, requires special care. Here we see that analysing the general UC (5.1) leads to further refinement. For a single factor $G \in K_{1}$, with $D \cdot S=2, D \cdot Y \geq 1$ only for $\mathrm{Sp}(1) \sim \mathrm{SU}(2)$ and $\mathrm{SU}(3)$.

We shall consider in greater detail the situation when UC leads to stronger constraints than KPC. Note that KPC $D \cdot Y \geq 0$ can be interpreted as the statement that the singular loci of the entire elliptically fibered $\mathrm{CY}_{3}$ are contained inside the degeneration loci of the elliptic fiber. We see that for $\mathrm{CY}$ threefolds with some special types of singularity structures, $\mathrm{UC}$ requires that $D \cdot Y \geq 1$, hence contains finer information about the residual divisor $Y$. In these cases, $\mathrm{UC}$ indicates that the singular loci of the elliptically fibered $\mathrm{CY}_{3}$ cannot sweep out the entire degeneration loci of the elliptic fiber.

Case 2. For a gauge group of type $K_{1}$, the vanishing orders for $f, g$ and $\Delta$ in Weierstrass data are given by $(0,0, m)$. Recall also that $(f, g, \Delta) \in(\Gamma(-4 K), \Gamma(-6 K), \Gamma(-12 K))$.

Let us assume that UC does not hold and we can take $Y$ to be numerically equivalent to 0 , i.e. have zero intersections with any curve in $B$. Then $-12 K=x_{1} S_{1}+x_{2} S_{2}$. Since $f$ and $g$ do not vanish along the gauge divisors $S_{1}$ and $S_{2}$, we have:

$$
S_{1} \cdot K=S_{2} \cdot K=0=K \cdot K .
$$

This implies

$$
x_{1} S_{1} \cdot S_{1}+x_{2} S_{1} \cdot S_{2}=x_{1} S_{1} \cdot S_{2}+x_{2} S_{2} \cdot S_{2}=0 .
$$

Using the correspondence between the geometric data and the coefficients of the anomaly polynomial (see section 5.1), we have ${ }^{25}$

$$
K \cdot K=0 \mapsto a \cdot a=9-n_{T}=0
$$

The adjunction formula yields:

$$
S_{1} \cdot S_{1}=2 g_{1}-2, \quad \text { and } \quad S_{2} \cdot S_{2}=2 g_{2}-2
$$

where $g_{1}$ ad $g_{2}$ are the genera of the corresponding curves.

\footnotetext{
${ }^{25}$ We will use the full set of standard anomaly cancellation conditions which can be found e.g. in [10].
} 
There are two possibilities:

A: divisors $S_{1}$ and $S_{2}$ are not in the same class. Since they are represented by curves, $S_{1} \cdot S_{2} \geq 0$. If $S_{1} \cdot S_{2}>0$, we obtain $S_{1} \cdot S_{1}<0$ and $S_{2} \cdot S_{2}<0$. Applying the adjunction formula finally yields $S_{1} \cdot S_{1}=S_{2} \cdot S_{2}=2 g_{1}-2=2 g_{2}-2=-2, S_{1} \cdot S_{2}=2$ and $x_{1}=x_{2}$. When $S_{1} \cdot S_{2}=0$, we obtain the same conditions as in the case B.

B: divisors $S_{1}$ and $S_{2}$ are in the same class. Then we have $g_{1}=g_{2}=1$ and $S_{1} \cdot S_{2}=0$.

For case A, let us analyse the example with gauge group $\mathrm{SU}(m) \times \mathrm{SU}(m)$ for illustration. ${ }^{26}$ Note that $x_{1}=x_{2}=m$. We also have $g_{1}=g_{2}=0$, hence the gauge divisor is given by a rational curve.

Using the anomaly cancellation conditions once more, we can see that in order to cancel the irreducible gauge anomaly $\sim \operatorname{tr} F_{i}^{4}, 2 m$ fundamental hypers are needed for each $\mathrm{SU}(m)$. The condition $S_{1} \cdot S_{2}=2$ tell that in fact there are 2 bifundamentals. To summarise, this case corresponds to a minimal supergravity theory with $n_{T}=9$, YM multiplets with $\mathrm{SU}(m) \times \mathrm{SU}(m)$ gauge group and two hypermultiplets in bifundamental. This example has appeared in [18]. Notice that the six-dimensional unitarity condition of [18] in this case is also stronger than KPC. Based on this it was conjectured that unitarity condition may teach us something about elliptically fibered $\mathrm{CY}_{3}$.

If $-12 K=m S_{1}+m S_{2}$ corresponds to an elliptic $\mathrm{CY}_{3}$, then $\mathrm{UC}$ can be violated by a $\mathrm{F}$ theory model. This does not seem to be very reasonable. Hence we should conclude if this supergravity is to be realised by an elliptic $\mathrm{CY}_{3}$, the effective divisor cannot be set numerically to zero. There necessarily should be extra contributions from non-singular (of the entire elliptic $\mathrm{CY}_{3}$ ) degeneration (of the elliptic fiber) loci which do not intersect $S_{1}$ or $S_{2}$ and are not detected by the low-energy spectrum.

For case $\mathrm{B}$, the analysis and conclusion would be the same as case $\mathrm{A}$ : $\mathrm{uC}$ requires the remaining effective divisor not numerically equivalent to 0 for corresponding elliptically fibered $\mathrm{CY}_{3}$ to exist.

Case 3. The divisor $Y=-12 K-x S$ cannot be numerically equivalent to 0 . Assuming it does, would lead to $-12 K=x S$. There will be a single gauge group $G$, and the number of the adjoint hypers is given by the genus of the corresponding curve $g_{S}$. This is not very constraining for the groups that have vanishing $\operatorname{tr} F^{4}$ (i.e. $E_{6,7,8}, G_{2}, F_{4}, \mathrm{SU}(2)$, $\mathrm{SU}(3)$ and $\mathrm{SO}(8))$. For all others the vanishing of the irreducible gauge anomaly requires a hypermultiplet in the adjoint, and hence $g_{S}=1$. Using the adjunction formula $S \cdot K+S \cdot S=$ $2 g_{S}-2$, one obtains

$$
S \cdot S-\frac{x}{12} S \cdot S=2 g_{S}-2=0
$$

- For $S \cdot S=0$, we have $K \cdot K=9-n_{T}=0$. As in Case 2, an analysis along the same leads to a similiar conclusion that UC is slightly stronger than KPC and hints at a hidden structure in the related elliptic $\mathrm{CY}_{3}$ (if it exists): the residual divisor $Y$

\footnotetext{
${ }^{26}$ We can further restrict to gauge group $\mathrm{SU}(6 m) \times \mathrm{SU}(6 m)$ or $\mathrm{SU}(6) \times \mathrm{Sp}(3)$ by a more refined analysis (see section 5.2.2).
} 
cannot be numerically 0. (A more detailed analysis in section 5.2.2 tells that UC is stronger than KPC only for $\mathrm{SU}(12 n)$ and $\mathrm{SU}(12 n-1))$.

- For $S \cdot S \neq 0$, eq. (5.20) gives $x=12$. According to table 1, this can only happen for $I_{12}$ and $I_{6}^{*}$ singularities. The corresponding gauge groups are $\mathrm{SU}(12), \mathrm{SO}(19)$, $\mathrm{SO}(20)$ and $\mathrm{Sp}(6)$. A detailed analysis, presented in the next section, leads to the conclusion that UC is stronger than KPC and hints at a finer information on the residual divisor $Y$.

- Finally we turn to gauge groups $E_{6,7,8}, G_{2}, F_{4}, \mathrm{SU}(2), \mathrm{SU}(3)$ and $\mathrm{SO}(8)$. For all of them $x<12$, and hence $D \cdot S=\frac{-12}{x} D \cdot K$ being an integer leads to $D \cdot S \geq 2$. As a result $(x-y) D \cdot S \geq 3$ and for these cases UC is weaker than KPC. For example in the $\mathrm{SU}(3)$ case we see $x=3,4$ (see table 1 ) and $D \cdot S=-4 D \cdot K$ and $-3 D \cdot K \geq 3$. Hence $(x-y) D \cdot S \geq 3$ as $x-y \geq 1$.

The further analysis of these special cases of gauge groups is presented in the next section where we study general nef divisors.

\subsubsection{General nef divisor $D$}

We can now turn to the general case, where we require only that $D$ is a nef divisor and analyse KPC and UC more carefully with a purpose of singling out the cases which KPC is satisfied coarsely (if we just ignore the effective divisor $Y=-12 K-\sum_{i} x_{i} S_{i}$ ) while UC is violated. These are the cases where UC should be revealing a hidden finer structure in the elliptic $\mathrm{CY}_{3}$ involved.

Let us start by collecting a slightly rewriting KPC and UC (in its general form, and not the strong form (5.8)):

$$
\begin{aligned}
& -12 D \cdot K=D \cdot Y+\sum_{i} x_{i} D \cdot S_{i} \\
& -12 D \cdot K \geq 3+\sum_{i} \frac{\operatorname{dim} G_{i}}{D \cdot S_{i}+h_{i}^{\vee}} D \cdot S_{i}=3+\sum_{i} \mu_{i} D \cdot S_{i}
\end{aligned}
$$

where as before $G_{i}$ is the (non-Abelian) gauge group hosted on (singular) gauge divisor $S_{i}$, and we have defined $\mu_{i}=\frac{\operatorname{dim} G_{i}}{D \cdot S_{i}+h_{i}^{\nabla}}$. When $D \cdot S_{i}=1, \mu_{i}=y_{i}$, otherwise $\mu_{i}<y_{i}$. Replacing $\mu_{i}$ by $y_{i}$ result in the strongest version of $\mathrm{UC}$ (as already notice, in some cases this strong version may fail, while the UC (5.8) actually holds).

Note that $-12 D \cdot K \in 12 \mathbb{Z}_{+}$, and hence:

$$
\begin{aligned}
\sum_{i} x_{i} D \cdot S_{i} & \leq D \cdot Y+\sum_{i} x_{i} D \cdot S_{i}=-12 D \cdot K=12 n \\
3+\sum_{i} \mu_{i} D \cdot S_{i} & \leq-12 D \cdot K=12 n
\end{aligned}
$$

for a positive integer $n$. It is not hard to see that there are three possibilities

- If for all gauge divisors, $D \cdot S_{i}=0$, the two conditions are equivalent trivially. This is very unlikely to happen in a base $B$. 
- If at least three gauge divisors $S_{1,2,3}$ have the property that $D \cdot S_{1,2,3}>0$ (this holds for a generic $S_{i}$ ), then even the strongest version of UC is weaker than KPC. The same conclusion holds for the case of at least two gauge divisors where at least one yields a $K_{2}$ type gauge group (due to $x_{i}-\mu_{i} \geq x_{i}-y_{i} \geq 2$ for $K_{2}$ type).

- For the remaining cases, let us notice that positive integer solution for $D \cdot S_{i}$ exist only when

$$
12 n-3<\sum_{i} \mu_{i} D \cdot S_{i} \leq 12 n-\sum_{i}\left(x_{i}-\mu_{i}\right) D \cdot S_{i}
$$

is satisfied. This condition implies that while KPC is respected, UC is violated. For these cases, UC can lead to stronger constraints than $\mathrm{KPC}$ and $Y=-12 K-\sum_{i} x_{i} S_{i}$ cannot be numerically 0 in order for F-theory models not to violate UC.

Obviously we are interested only in the last situation, where we can divide the nontrivial solutions of condition (5.23) into two cases:

- There are two gauge divisors $S_{1}$ and $S_{2}$ in gauge groups of type $K_{1}$, and $D \cdot S_{1,2}>0$. The cases are where UC is more constraining than KPC are:

- $\mathrm{SU}(n) \times \mathrm{SU}(m)$ with $m+n \in 12 \mathbb{Z}$ and $D \cdot S_{1,2}=1$.

- $\mathrm{SU}(12 n-2) \times \mathrm{Sp}(1), \mathrm{SU}(12 n-4) \times \mathrm{Sp}(2)$ and $\mathrm{SU}(12 n-6) \times \mathrm{Sp}(3)$ with $D \cdot S_{1,2}=1$. Here $\operatorname{Sp}(1), \operatorname{Sp}(2)$ and $\operatorname{Sp}(3)$ should come from $I_{2}, I_{4}$ and $I_{6}$ type singularities (see table 1).

For these gauge groups we need to impose a further condition on the effective divisor $D \cdot Y \geq 1$ in order for UC not be violated by F-theory models.

- There is only a single gauge divisor $S$ with the property $D \cdot S>0$. UC can be more constraining than KPC only for the gauge groups

- $\mathrm{SU}(12 n), \mathrm{SU}(12 n-1)$ with $D \cdot S=1$.

- $\mathrm{SO}(24 n-5), \mathrm{SO}(24 n-4)$ and $\mathrm{Sp}(6 n)$ with $D \cdot S=1$. Here $\mathrm{Sp}(6 n)$ should come from $I_{12 n}$ type singularity (see table 1 ).

In all these cases $\mu_{i}=y_{i}$. Only when the divisor $Y=-12 K-\sum_{i} x_{i} S_{i}$ satisfies $D \cdot Y \geq 2$ for $\mathrm{SU}(12 n-1)$ and $D \cdot Y \geq 1$ for the rest, UC is not violated in F-theory models.

For completeness, we can present an example of a group where no extra constraints emerge. For $E_{6}$, UC would be stronger than KPC only if we have a solution for

$$
12 n-3<\mu_{E_{6}} D \cdot S_{E_{6}} \leq 12 n-\left(x_{E_{6}}-\mu_{E_{6}}\right) D \cdot S_{E_{6}} .
$$

This would require

$$
3>\left(x_{E_{6}}-\mu_{E_{6}}\right) D \cdot S_{E_{6}} \geq\left(x_{E_{6}}-y_{E_{6}}\right) D \cdot S_{E_{6}}=2 D \cdot S_{E_{6}} \rightarrow D \cdot S_{E_{6}}=1
$$

leading to $\mu_{E_{6}}=y_{E_{6}}=6$. However, then $12 n-3<\mu_{E_{6}} D \cdot S_{E_{6}} \leq 12 n-\left(x_{E_{6}}-\mu_{E_{6}}\right) D \cdot S_{E_{6}}$ becomes $12 n-3<6 \leq 12 n-2$ which doesn't have a solution! Similar arguments can be applied to other cases. 


\subsubsection{Special cases where UC is stronger than KPC}

Following the discussion in the previous section, we can give a precise statement about what UC may teach us about elliptic Calabi-Yau threefolds through F-theory models:

For F-theory on an elliptic $\mathrm{CY}_{3}$ over base $B$, only when there exist gauge (singular) divisors $\left\{S_{i}\right\}$ and a nef divisor $D$ on the base $B$, which satisfy some (very special) numerical conditions, UC hints at a finer information than contained in KPC, on the effective divisor $Y=-12 K-x_{i} S_{i}$.

There are three types of models where this can happen:

A: There exists one gauge divisor $S_{1} \in\left\{S_{i}\right\}$ hosting a gauge group SU(12n) or SU(12n1 ), and a nef divisor $D$ satisfying $D \cdot S_{1}=1$ and $D \cdot S_{i}=0$ for all other $i \neq 1$, as well as $-D \cdot K \in \mathbb{Z}_{+}$. Then such a nef divisor $D$ should satisfy $D \cdot Y \geq 1$ for $\operatorname{SU}(12 n)$ and $D \cdot Y \geq 2$ for $\mathrm{SU}(12 n-1)$ in order for UC to be satisfied by F-theory models

B: There exists one gauge divisor $S_{1} \in\left\{S_{i}\right\}$ hosting a gauge group $\mathrm{SO}(24 n-5), \mathrm{SO}(24 n-$ 4) or $\mathrm{Sp}(6 n)$ (which comes from $I_{12 n}$ type singularity), and a nef divisor $D$ satisfying $D \cdot S_{1}=1$ and $D \cdot S_{i}=0$ for all $i \neq 1$, as well as $-D \cdot K \in \mathbb{Z}_{+}$. Then such a nef divisor $D$ should satisfy $D \cdot Y \geq 1$ in order for UC to be satisfied by F-theory models

C: There exist two gauge divisors $S_{1}, S_{2} \in\left\{S_{i}\right\}$ hosting gauge group $\mathrm{SU}(a) \times \mathrm{SU}(12 n-a)$, $\mathrm{Sp}(1) \times \mathrm{SU}(12 n-2), \mathrm{Sp}(2) \times \mathrm{SU}(12 n-4)$ or $\mathrm{SU}(12 n-6) \times \mathrm{Sp}(3)($ where $\mathrm{Sp}(1), \mathrm{Sp}(2)$ and $\operatorname{Sp}(3)$ come from $I_{2}, I_{4}$ and $I_{6}$ type singularities) and a nef divisor $D$ satisfying $D \cdot S_{1,2}=1$ and $D \cdot S_{i}=0$ for all $i \neq 1,2$, as well as $-D \cdot K \in \mathbb{Z}_{+}$. Then such a nef divisor $D$ should satisfy $D \cdot Y \geq 1$ in order for UC to be satisfied by F-theory models

Note that an example in class $\mathrm{C}$ has been discussed in section 5.2.4, and has appeared previously in [18], where it was pointed out that (six-dimensional) unitarity condition can lead to stronger constraints than KPC.

To conclude, in a generic F-theory model UC leads to weaker constraints than KPC. Under some special conditions UC hints at finer information about the possible elliptic $\mathrm{CY}_{3}$ than $\mathrm{KPC}$ on the remaining effective divisor $Y=-12 K-\sum_{i} x_{i} S_{i}$ on the base.

In the next section we shall briefly discuss some examples, but we finish this section with some remarks.

- In all special cases, where UC is stronger than KPC, the numerical constraints on the gauge divisors and on the residual divisor $Y$ are rather strong. We have not studied if and how many non-trivial realisations of these conditions exist in elliptic CY threefolds.

- A general lesson provided by UC in all above special cases for elliptic $\mathrm{CY}_{3}$ is that the residual divisor $Y$ on the base of the elliptic $\mathrm{CY}_{3}$ should have nontrivial numerical properties and not be numerically equivalent to 0 , and in general the gauge divisors do not sweep out the entire $-12 K$.

- All cases where UC is stronger than KPC involve at most two gauge divisors (of fixed type) intersecting the nef divisor $D$. The corresponding supergravity models 
can however contain more than two gauge factors. The extra gauge groups should come from singular divisors that do not intersect $D$.

- Only in one special case, 6d UC in [18] is stronger than the 5d UC discussed in this paper. This happens when an additional condition $D \cdot D+D \cdot K=-2$ is satisfied, and the nef divisor $D$ is a genus 0 curve. For this very special situation, we need to decrease the upper bound of UC by $1 .{ }^{27}$

- Further compactification on a circle to four dimensions does not lead to further unitarity constraints.

\subsubsection{Examples}

In order to illustrate the previous discussion, we may consider three examples of elliptic $\mathrm{CY}_{3}$ which are fibrations over Hirzebruch surfaces $\mathbb{F}_{n}$ (the details of the geometry of these examples can be found in e.g. [10]). For all these examples, we shall see that the residual divisor $Y$ is indeed numerically nontrivial (its intersections with all nef divisors are strictly positive). Our forth example has already appeared in the text and in [18], and, to the best of our knowledge, has no known F-theoretic realisation. We shall see that if such realisation exists, it would require $Y$ to be numerically nontrivial.

First we collect some data on $\mathbb{F}_{n}$, which will be useful in the first three examples. The effective divisor is spanned by $D_{v}$ and $D_{s}$. Their intersection data are

$$
D_{v} \cdot D_{v}=-m, \quad D_{v} \cdot D_{s}=1, \quad D_{s} \cdot D_{s}=0
$$

and the canonical divisor $K$ satisfies

$$
-12 K=24 D_{v}+12(m+2) D_{s} .
$$

Example 1. 6d supergravity with a single $\mathrm{SU}(N)$ can be modelled on base $\mathbb{F}_{2}$. These types of F-theory models have some overlap with special case A in section 5.2.3. In these cases, gravity anomaly cancellation requires $N \leq 15$. The gauge divisor on $\mathbb{F}_{2}$ is $S=D_{v}$, and the residual effective divisor $Y$ is given by

$$
Y=-12 K-N D_{v}=(24-N) D_{v}+48 D_{s}
$$

Hence any nef divisor $D$ with $D \cdot D_{v}>0$ has to have the form $\alpha D_{v}+\beta D_{s}$ for some integers $\alpha$ and $\beta$ with $\beta>2 \alpha$. Requiring in addition that $D \cdot K<0$ and $D \cdot D \geq 0$ yields $\alpha \geq 0, \beta>0$. So any such nef divisor will have $D \cdot Y=(24-N) \beta+2 N \alpha \geq 9$.

\footnotetext{
${ }^{27} \mathrm{As}$ in the case when the nef divisor is a rational curve, it is not hard to see from (4.11) that the upper bound set by $6 \mathrm{~d} \mathrm{UC}$ is $-12 D \cdot K-4$ rather than $-12 D \cdot K-3$ set by $5 \mathrm{~d}$ UC. In this case, UC is stronger than KPC only when there exist positive integer solutions for $D \cdot S_{i}$ satisfying $12 n-4<\sum_{i} \mu_{i} D \cdot S_{i} \leq$ $12 n-\sum_{i}\left(x_{i}-\mu_{i}\right) D \cdot S_{i}$. As a result, since the lower bound is relaxed by 1 when compared with (5.23), some new special cases will appear. The conclusion will still be the same: for these cases $Y=-12 K-\sum_{i} x_{i} S_{i}$ should satisfy some numerical properties in order for F-theory models not to violate UC.
} 
Example 2. 6d supergravity with gauge group $\mathrm{SO}(16) \times \mathrm{SU}(4) \times \mathrm{SU}(4)$ is modelled over $\mathbb{F}_{4}$. The gauge divisors are $S_{1}=D_{v}, S_{2}=D_{v}+4 D_{s}$ and $S_{3}=D_{v}+8 D_{s}$. The residual effective divisor is given by:

$$
Y=-12 K-10 D_{v}-4\left(D_{v}+4 D_{s}\right)-4\left(D_{v}+8 D_{s}\right)=6 D_{v}+24 D_{s}
$$

It is not hard to see that the conditions $\alpha \geq 0, \beta>0, \beta \geq 2 \alpha$ are required in order for any divisor $D=\alpha D_{v}+\beta D_{s}$ to satisfy $D \cdot D \geq 0, D \cdot K<0$ and $D \cdot S_{i} \geq 0$. As a result, $D \cdot Y=6 \beta \geq 6$.

Example 3. 6d supergravity with gauge group $\mathrm{U}(1) \times \mathrm{SU}(8)$ is modelled on $\mathbb{F}_{0}$. For our purposes, we can ignore the Abelian factor. The relevant gauge divisor is $S=2 D_{v}+2 D_{s}$, and the residual effective divisor $Y$ is given by

$$
Y=-12 K-8\left(2 D_{v}+2 D_{s}\right)=8 D_{v}+8 D_{s}
$$

Any nef divisor $D=\alpha D_{v}+\beta D_{s}$ satisfies $D \cdot K<0, D \cdot\left(2 D_{v}+2 D_{s}\right) \geq 0, D \cdot D \geq 0$ only provided that $\alpha+\beta>0$ and $\alpha \beta \geq 0$. As a result, $D \cdot Y=8 \alpha+8 \beta \geq 8$. Notice that this theory passes the unitarity test even with the additional $\mathrm{U}(1)$ included (since any $\mathrm{U}(1)$ factor can only contribute 1 to the central charge).

UC and KPC criteria can also be translated to conditions on some physical data of general $6 \mathrm{~d}$ anomaly free minimal supergravity without knowing whether it can be realised in F-theory or not. The following is one example of applying UC and KPC to a $6 \mathrm{~d}$ anomaly free minimal supergravity model.

Example 4. 6d supergravity with $\mathrm{SU}(N) \times \mathrm{SU}(N)$ with two bifundamentals and 9 tensor multiplets is an anomaly free theory [18]. The relevant data (the constant vectors in the GS couplings) are given by: ${ }^{28}$

$$
\begin{array}{ll}
\Omega=\operatorname{diag}\left(+1,(-1)^{9}\right), & a=\left(-3,(+1)^{9}\right) \\
b_{1}=\left(1,-1,-1,-1,0^{6}\right), & b_{2}=\left(2,0,0,0,(-1)^{6}\right) .
\end{array}
$$

Choosing the string charge as $Q=(1,0,0,0,-1,0 \ldots, 0)$, we obtain $Q \cdot Q=0, Q \cdot a=-2$ and $Q \cdot b_{1}=Q \cdot b_{2}=1$. The unitarity condition (5.8) gives us:

$$
2(N-1) \leq 24-3
$$

Note that in this case $Q \cdot Q+Q \cdot a=-2$, and thus the 6 d unitarity condition of [18] is slightly stronger than $5 \mathrm{~d}$ UC: a shift by 1 on right hand side is needed and $2(N-1) \leq 24-4$. Either way, the bound is $N \leq 11$, while the Kodaira positivity condition yields:

$$
2 N \leq 24 \rightarrow N \leq 12 .
$$

\footnotetext{
${ }^{28}$ Note that the string charge here is different from the one used in [18]. The choice of $Q$ in [18] leads to $Q \cdot K=Q \cdot Q=-1$ and the putative corresponding divisor $D$ on the base $B$ of elliptic Calabi-Yau side would no longer be nef.
} 
We see that in this case $\mathrm{UC}$ is slightly stronger than $\mathrm{KPC}$. Also notice that $\mathrm{SU}(12) \times \mathrm{SU}(12)$ case, which satisfies KPC but violates UC, belongs to case C, enumerated in section 5.2.3.

Assuming that this theory has an F-theoretic realisation and that there is an underlying elliptic $\mathrm{CY}_{3}$, the residual effective divisor would be

$$
-12 K=N S_{1}+N S_{2}+Y
$$

Restricting for simplicity to the case $N \geq 4$, the singular divisors are of type $I_{N}$ (see table 1). ${ }^{29}$ Then we have $S_{1} \cdot K=S_{2} \cdot K=0$. Since there are two hypers in the bifundamental and no hypers in the adjoint, we should take $S_{1} \cdot S_{1}=-2=S_{2} \cdot S_{2}$ and $S_{1} \cdot S_{2}=2$, while $n_{T}=9$ translates into $K \cdot K=0$. If we just follow KPC and set the residual effective divisor $Y$ to be numerically 0 , we get exactly case A which we discussed in section 5.2.1. Based on the above discussion, we can see that the new lesson UC offered in this case is that the relation

$$
-12 K=12 S_{1}+12 S_{2}
$$

cannot be realised on the base $B$ of an elliptic Calabi-Yau threefold with the required singularity structure, and the residual effective divisor $Y=-12 K-12 S_{1}-12 S_{2}$ has to be numerically non-trivial.

\section{Acknowledgments}

We are grateful to Federico Bonetti, Hadi Godazgar, Thomas Grimm, Ilarion Melnikov, Ioannis Papadimitriou, Sergei Kuzenko and Piljin Yi for interesting conversations and correspondence. The work RM is supported in part by ERC Grant 787320-QBH Structure and by ERC Grant 772408-Stringlandscape.

\section{A Reduced anomalies and Bardeen-Zumino counterterms}

To prove equation 2.5, we first recall the general descent procedure (cf. [41] for review). For this it is sufficient to consider anomaly polynomials of the form

$$
I_{2 n+2}=P\left(F^{n+1}\right)
$$

where $P$ is a invariant symmetric polynomial of order $n+1$. Then one can write $I_{2 n+2}=$ $d I_{2 n+1}^{0}$ where

$$
I_{2 n+1}^{0}=(n+1) \int_{0}^{1} d t P\left(A, F_{t}^{n}\right)
$$

where $F_{t}=t F+\left(t^{2}-t\right) A^{2}$. From this one finds $\delta_{\epsilon} I_{2 n+1}^{0}=d I_{2 n}^{1}$ with

$$
I_{2 n}^{1}=n(n+1) \int_{0}^{1} d t(t-1) P\left(d \epsilon, A, F_{t}^{n-1}\right)
$$

\footnotetext{
${ }^{29}$ We explude $\mathrm{SU}(2)$ and $\mathrm{SU}(3)$ gauge groups since they may also be realised by $I I I, I V$-type singularities.
} 
which solves the Wess-Zumino consistency condition, i.e. it is a possible (consistent) anomaly in $2 n$ dimensions. The Bardeen-Zumino polynomial [17], which was constructed to modify the current in order to satisfy a covariant anomalous conservation law (i.e. covariant anomaly), is defined $\operatorname{as}^{30}$

$$
\phi \cdot X=n(n+1) \int_{0}^{1} d t t P\left(\phi, A, F_{t}^{n-1}\right) .
$$

This is a $2 n-1$ form. We need its gauge variation. This is straightforward to work out using $\delta A=d \epsilon+[A, \epsilon]$ and $\delta \phi=[\phi, \epsilon]$, with the result

$$
n(n+1) \int_{0}^{1} d t\left\{t P\left(d \epsilon, \phi, F_{t}^{n-1}\right)+t^{2}(t-1)(n-1) P\left(A, \phi,\{d \epsilon, A\}, F_{t}^{n-2}\right)\right\} .
$$

We have to compare this with the compactified anomaly, i.e. with

$$
n(n+1) \int_{0}^{1} d t(t-1) \int_{S^{1}} P\left(d \epsilon, \hat{A}, \hat{F}_{t}^{n-1}\right)
$$

where the hatted quantities are in $2 n$ dimensions while the unhatted ones are in $2 n-1$ dimensions:

$$
\hat{A}=A+\varphi \quad \varphi=\phi d y \quad \int_{S^{1}} \varphi=\phi
$$

and

$$
\hat{F}=F+d \varphi+\{A, \varphi\}, \quad \hat{F}_{t}=F_{t}+t d \varphi+t^{2}\{A, \varphi\}
$$

$y$ is the compact coordinate, $A$ is a one-form, $\phi$ and $\epsilon$ are zero-form in $2 n-1$ dimensions.

In (A.6) only the piece linear in $\varphi$ survives the integration over $y$ (we moved $d y$ all the way to the right):

$$
\int_{S^{1}} P\left(d \epsilon, \hat{A}, \hat{F}^{n-1}\right)=P\left(d \epsilon, \phi, F_{t}^{n-1}\right)+(n-1) P\left(d \epsilon, A, t d \phi+t^{2}[A, \phi], F_{t}^{n-2}\right) .
$$

We first consider the term $t P\left(d \epsilon, A, d \phi, F_{t}^{n-2}\right)$. Using

$$
d P\left(d \epsilon, A, \phi, F_{t}^{n-2}\right)=-P\left(d \epsilon, d A, \phi, F_{t}^{n-2}\right)+P\left(d \epsilon, A, d \phi, F_{t}^{n-2}\right)+P\left(d \epsilon, A, \phi, d F_{t}^{n-2}\right)
$$

and

$$
d F_{t}^{n-2}=(n-2) d F_{t} F_{t}^{n-3}=(n-2) t\left[F_{t}, A\right] F_{t}^{n-3}
$$

which is valid inside $P$ we obtain, up to a total derivative, ${ }^{31}$

$$
t P\left(d \epsilon, A, d \phi, F_{t}^{n-2}\right)=t P\left(d \epsilon, d A, \phi, F_{t}^{n-2}\right)+(n-2) t^{2} P\left(d \epsilon, A, \phi,\left[A, F_{t}\right], F_{t}^{n-3}\right) .
$$

To eliminate the last term, use the invariance of $P$, i.e.

$$
\begin{aligned}
0= & P\left(\{A, d \epsilon\}, A, \phi, F_{t}^{n-2}\right)-P\left(d \epsilon,\{A, A\}, \phi, F_{t}^{n-2}\right) \\
& +P\left(d \epsilon, A,[A, \phi], F_{t}^{n-2}\right)+P\left(d \epsilon, A, \phi,\left[A, F_{t}^{n-2}\right]\right)
\end{aligned}
$$

\footnotetext{
${ }^{30}$ In [17] BZ used a one form $B$ instead of $\phi$.

${ }^{31}$ All expressions should be understood to be valid up to a total derivative in $M_{2 n-1}$.
} 
Collecting terms we find

$$
\begin{aligned}
\int_{S^{1}} P\left(d \epsilon, \hat{A}, \hat{F}_{t}^{n-1}\right)= & P\left(d \epsilon, \phi, F_{t}^{n-1}\right)-t^{2}(n-1) P\left(\{A, d \epsilon\}, A, \phi, F_{t}^{n-1}\right) \\
& +t(n-1) P\left(d \epsilon, d A+2 t A^{2}, \phi, F_{t}^{n-2}\right) .
\end{aligned}
$$

The commutator term in (A.9) has cancelled. Using $d A+2 t A^{2}=\frac{d}{d t} F_{t}$ this becomes

$$
P\left(d \epsilon, \phi, F_{t}^{n-1}\right)-t^{2}(n-1) P\left(\{A, d \epsilon\}, A, \phi, F_{t}^{n-2}\right)+t \frac{d}{d t} P\left(d \epsilon, \phi, F_{t}^{n-1}\right) .
$$

We need to multiply this by $n(n+1)(t-1)$ and integrate over $t$. Collecting terms, which involves a partial integration where the boundary terms vanish identically, one finally finds

$$
\begin{aligned}
& \int_{S^{1}} I_{2 n}^{1}(\epsilon, \hat{A}, \hat{F}) \\
& \quad=n(n+1) \int_{0}^{1} d t\left\{t P\left(d \epsilon, \phi, F_{t}^{n-1}\right)+t^{2}(t-1)(n-1) P\left(\{A, d \epsilon\}, A, \phi, F_{t}^{n-2}\right)\right\}
\end{aligned}
$$

which is the same as (A.5) and therefore completes the proof.

The above proof holds when the gauge group $G$ is the same in $2 n$ and $2 n-1$ dimensions, and in principle can be generalised to the case when Wilson lines are turned on. For the gravitational anomalies, this is always the case since upon reduction $\operatorname{Diff}\left(M_{2 n}\right)$ is reduced to $\operatorname{Diff}\left(M_{2 n-1}\right)$. We shall not attempt general proofs here and simply consider the mixed $\mathrm{U}(1)$-gravitational anomaly in $d=4$. Starting from a six-form $I_{6}=\hat{F} \wedge \operatorname{tr}(\hat{R} \wedge \hat{R})$ descending along the $\mathrm{U}(1)$ leads to an anomaly given by

$$
I_{4}^{1}=\epsilon \operatorname{tr}(\hat{R} \wedge \hat{R})
$$

$\epsilon$ is as before the gauge variation parameter.

We can now explicitly compute the reduction of $I_{4}^{1}$ along a circle, by assuming there is isometry direction and all the fields depend on three coordinates only. ${ }^{32}$ Denoting the KK vector by $A^{0}$ and its curvature two-form by $T,{ }^{33}$ the Wilson line is given by

$$
\Phi_{\alpha \beta}=-\frac{1}{2} T_{\alpha \beta}
$$

where $\alpha, \beta$ are the three-dimensional tangent space indices. It can be verified explicitly that

$$
\operatorname{tr}\left(\Phi X_{\mathrm{BZ}}\right)=2 A \wedge\left(\hat{R}^{\alpha \beta} \Phi_{\alpha \beta}\right)
$$

where the three-dimensional component of the curvature two-form is given by $\hat{R}^{\alpha \beta}=$ $R^{\alpha \beta}-\frac{1}{4} T^{\alpha} T^{\beta}-\frac{1}{2} T^{\alpha \beta} T-\frac{1}{2} D T^{\alpha \beta} A^{0}$. Moreover

$$
\operatorname{tr}\left(\Phi X_{\mathrm{BZ}}\right)-\int_{S^{1}} I_{4}^{1}=\frac{1}{4} A \wedge d\left(\left(T^{\alpha \beta} T_{\alpha \beta}\right) A^{0}\right) .
$$

\footnotetext{
${ }^{32}$ For simplicity we just consider here the Lorentz anomaly and the reduction from $\mathrm{SO}(3,1)$ to $\mathrm{SO}(2,1)$.

${ }^{33}$ The curvature two-form of the $\mathrm{U}(1)$ fibrations $T \in H^{2}\left(M_{2 n-1}, \mathbb{Z}\right)$ can be written using the threedimensional vielbeins as $T=d A^{0}=\frac{1}{2} T_{\alpha \beta} e^{\alpha} \wedge e^{\beta}$. We shall treat $T_{\alpha \beta}$ as an $\mathrm{SO}(2,1)$-valued zero-form and also use a one-form $T^{\alpha}=T_{\beta}^{\alpha} e^{\beta}$.
} 
This term is covariant both under the $\mathrm{U}(1)$ gauge transformation and the $3 \mathrm{~d}$ Lorentz gauge transformation $\mathrm{SO}(2,1)$. Furthermore, as one should expect, this term is not covariant under the $\mathrm{U}(1)$ gauge transformation of the KK vector $A^{0}$. This is due to the compactification Ansatz explicitly breaking this part of gauge symmetry inherited from the $4 \mathrm{~d}$ Lorentz gauge symmetry $\mathrm{SO}(3,1)$.

This calculation can be extended to arbitrary dimensions (including the case of purely gravitational anomalies in $2 n+2$ dimensions). The general formula will always contain a KK U(1) non-covariant part. This is the part denoted by ellipsis in (1.1).

\section{B Alternative proof that strings with $Q \cdot Q>0$ and $Q \cdot b_{i}>0$ are supergravity strings}

Here we present an alternative proof of the statement that a $6 \mathrm{~d}$ string with charge $Q$ which satisfies $Q \cdot Q>0$ and $Q \cdot b_{i}>0$ is a 5 d supergravity string after compactifying to $5 \mathrm{~d}$ on a circle. Or, in other words, those strings do not become tensionless on the entire Kähler moduli space.

First we identify the Kähler moduli $\mathcal{K}$ of $6 \mathrm{~d} \mathcal{N}=1$ supergravity. Recall that the BPS charges of the solitonic strings live on an integral lattice $\Lambda_{\mathrm{BPS}}$ of signature $\left(1, n_{T}\right)$. The Kähler moduli can then be defined as

$$
\mathcal{K}=\left\{j \in \Lambda_{\mathrm{BPS}} \otimes_{\mathbb{Z}} \mathbb{R} \mid j \cdot j=1, j \cdot a<0, j \cdot b_{i}>0\right\}
$$

The scalar $j \cdot j$ belongs to the universal hypermultiplet and since we study the tensor branch, we set $j \cdot j=1$. The condition $j \cdot b_{i}>0$ is required to have positive kinetic terms for the Yang-Mills action. For the supergravity theories with their origin in $F$-theory compactifications, the requirement $j \cdot a<0$ translates to a necessary requirement that the total space of the fibration is $\mathrm{CY}_{3}$. In general supergravity theories the justification of this condition is a bit more speculative.

We will use the notation $\mathcal{C K}$ to denote the cone over $\mathcal{K}$, i.e. when extending $\mathcal{K}$ to $\mathcal{C K}$, we replace the condition $j \cdot j=1$ by $j \cdot j>0$. In the F-theory context, this corresponds to the Kähler cone of the base, i.e. to those Kähler forms which lead to good $6 \mathrm{~d}$ supergravity theories.

Next, we choose a lattice point $Q$ inside $\mathcal{C K}$. As a result, a BPS string with charge vector $Q$ satisfies

$$
Q \cdot b_{i}>0 \quad Q \cdot a<0 .
$$

Since $Q$ is inside $\mathcal{C} \mathcal{K}$, one can show that in the Kähler moduli space $\mathcal{K}$, i.e. in the region $j \cdot j=1$, we always have

$$
Q \cdot j>0
$$

Indeed, since $Q$ is a lattice point inside $\mathcal{C K}$, we have

$$
Q \cdot Q=m>0 \quad \rightarrow \quad \frac{Q}{\sqrt{m}} \in \mathcal{K} .
$$


Since $j \in \mathcal{K}$, we can always write

$$
j=\sum_{i} r_{i} E_{i}, \quad r_{i} \geq 0
$$

Here $E_{i}$ are lattice points on the boundary of $\overline{\mathcal{C K}}$, the closure of $\mathcal{C K}$. Due to the condition $j \cdot j=1$, not all $r_{i} \geq 0$ can vanish simultaneously.

Now, since $\frac{Q}{\sqrt{m}} \in \mathcal{K}$ is a point not on the boundary of $\mathcal{K}$, we have that for every $i$

$$
\frac{Q}{\sqrt{m}} \cdot E_{i}>0
$$

is strictly positive. As not all $r_{i}$ can approach 0 at the same time even at the boundary of the Kähler moduli space (recall $j \cdot j=1$ ), we find that

$$
j \cdot Q=\sum_{i} \sqrt{m} r_{i} \frac{Q}{\sqrt{m}} \cdot E_{i}>0
$$

is strictly positive on the closed Kähler moduli space $\overline{\mathcal{K}}$. Therefore, the corresponding BPS string is a supergravity string after compactification on a circle, due to the fact that it cannot become tensionless at any point on the entire closed Kähler moduli space.

With all our conditions satisfied, we are describing the set of strings $\mathcal{S}$ with the following charges:

$$
\mathcal{S}=\left\{\mathcal{S}_{Q} \mid Q \in \Lambda_{\mathrm{BPS}}, Q \cdot Q>0, Q \cdot a<0, Q \cdot b_{i}>0\right\}
$$

If we compare it with

$$
\mathcal{C K}=\left\{j \in \Lambda_{\mathrm{BPS}} \otimes_{\mathbb{Z}} \mathbb{R} \mid j \cdot j>0, j \cdot a<0, j \cdot b_{i}>0\right\}
$$

we see these string charges are exactly the lattice points inside the cone $\mathcal{C K}$.

As a result, since the BPS strings with $Q \cdot Q>0$ and $Q \cdot b_{i}>0$ cannot go tensionless on the entire closed Kähler moduli space $\overline{\mathcal{K}}$, they belong to the set of supergravity strings after compactfication on a circle, and hence cannot be decoupled from gravity consistently.

Open Access. This article is distributed under the terms of the Creative Commons Attribution License (CC-BY 4.0), which permits any use, distribution and reproduction in any medium, provided the original author(s) and source are credited.

\section{References}

[1] C. Vafa, Evidence for F-theory, Nucl. Phys. B 469 (1996) 403 [hep-th/9602022] [InSPIRE].

[2] D.R. Morrison and C. Vafa, Compactifications of F-theory on Calabi-Yau threefolds. 1, Nucl. Phys. B 473 (1996) 74 [hep-th/9602114] [INSPIRE].

[3] D.R. Morrison and C. Vafa, Compactifications of F-theory on Calabi-Yau threefolds. 2, Nucl. Phys. B 476 (1996) 437 [hep-th/9603161] [INSPIRE]. 
[4] A.C. Cadavid, A. Ceresole, R. D'Auria and S. Ferrara, Eleven-dimensional supergravity compactified on Calabi-Yau threefolds, Phys. Lett. B 357 (1995) 76 [hep-th/9506144] [INSPIRE].

[5] S. Ferrara, R.R. Khuri and R. Minasian, $M$ theory on a Calabi-Yau manifold, Phys. Lett. B 375 (1996) 81 [hep-th/9602102] [INSPIRE].

[6] S. Ferrara, R. Minasian and A. Sagnotti, Low-energy analysis of $M$ and $F$ theories on Calabi-Yau threefolds, Nucl. Phys. B 474 (1996) 323 [hep-th/9604097] [InSPIRE].

[7] A. Grassi and D.R. Morrison, Anomalies and the Euler characteristic of elliptic Calabi-Yau threefolds, Commun. Num. Theor. Phys. 6 (2012) 51 [arXiv:1109.0042] [INSPIRE].

[8] V. Kumar, D.R. Morrison and W. Taylor, Global aspects of the space of $6 D N=1$ supergravities, JHEP 11 (2010) 118 [arXiv:1008.1062] [INSPIRE].

[9] S. Monnier and G.W. Moore, Remarks on the Green-Schwarz terms of six-dimensional supergravity theories, Commun. Math. Phys. 372 (2019) 963 [arXiv:1808.01334] [InSPIRE].

[10] W. Taylor, TASI lectures on supergravity and string vacua in various dimensions, arXiv:1104.2051 [INSPIRE].

[11] T. Weigand, F-theory, PoS(TASI2017) 016 [arXiv: 1806.01854] [INSPIRE].

[12] E. Poppitz and M. Ünsal, Index theorem for topological excitations on $R^{3} \times S^{1}$ and Chern-Simons theory, JHEP 03 (2009) 027 [arXiv: 0812 .2085] [INSPIRE].

[13] F. Bonetti, T.W. Grimm and S. Hohenegger, One-loop Chern-Simons terms in five dimensions, JHEP 07 (2013) 043 [arXiv: 1302.2918] [INSPIRE].

[14] F. Bonetti, T.W. Grimm and S. Hohenegger, Exploring $6 D$ origins of $5 D$ supergravities with Chern-Simons terms, JHEP 05 (2013) 124 [arXiv:1303.2661] [INSPIRE].

[15] P. Corvilain, T.W. Grimm and D. Regalado, Chiral anomalies on a circle and their cancellation in F-theory, JHEP 04 (2018) 020 [arXiv: 1710.07626] [INSPIRE].

[16] P. Corvilain, $6 d \mathcal{N}=(1,0)$ anomalies on $S^{1}$ and F-theory implications, JHEP 08 (2020) 133 [arXiv: 2005.12935] [INSPIRE].

[17] W.A. Bardeen and B. Zumino, Consistent and covariant anomalies in gauge and gravitational theories, Nucl. Phys. B 244 (1984) 421.

[18] H.-C. Kim, G. Shiu and C. Vafa, Branes and the Swampland, Phys. Rev. D 100 (2019) 066006 [arXiv: 1905.08261] [INSPIRE].

[19] S. Katz, H.-C. Kim, H.-C. Tarazi and C. Vafa, Swampland constraints on $5 d \mathcal{N}=1$ supergravity, JHEP 07 (2020) 080 [arXiv:2004.14401] [INSPIRE].

[20] H.-C. Tarazi and C. Vafa, On the finiteness of 6d supergravity Landscape, arXiv:2106.10839 [INSPIRE].

[21] C. Closset, T.T. Dumitrescu, G. Festuccia, Z. Komargodski and N. Seiberg, Contact terms, unitarity, and F-maximization in three-dimensional superconformal theories, JHEP 10 (2012) 053 [arXiv: 1205.4142] [INSPIRE].

[22] C. Closset, T.T. Dumitrescu, G. Festuccia, Z. Komargodski and N. Seiberg, Comments on Chern-Simons contact terms in three dimensions, JHEP 09 (2012) 091 [arXiv:1206.5218] [INSPIRE]. 
[23] D.L. Jafferis, The exact superconformal R-symmetry extremizes Z, JHEP 05 (2012) 159 [arXiv: 1012.3210] [INSPIRE].

[24] S.S. Pufu, The F-Theorem and F-Maximization, J. Phys. A 50 (2017) 443008 [arXiv: 1608.02960] [INSPIRE].

[25] D. Freed, J.A. Harvey, R. Minasian and G.W. Moore, Gravitational anomaly cancellation for M-theory five-branes, Adv. Theor. Math. Phys. 2 (1998) 601 [hep-th/9803205] [INSPIRE].

[26] J.A. Harvey, R. Minasian and G.W. Moore, Non-Abelian tensor multiplet anomalies, JHEP 09 (1998) 004 [hep-th/9808060] [INSPIRE].

[27] M.R. Gaberdiel and R. Gopakumar, Large $N=4$ holography, JHEP 09 (2013) 036 [arXiv: 1305.4181] [INSPIRE].

[28] J.M. Maldacena, A. Strominger and E. Witten, Black hole entropy in M-theory, JHEP 12 (1997) 002 [hep-th/9711053] [inSPIRE].

[29] A. Dabholkar and S. Nampuri, Quantum black holes, Lect. Notes Phys. 851 (2012) 165 [arXiv: 1208.4814] [INSPIRE].

[30] M. Dunajski and S.A. Hartnoll, Einstein-Maxwell gravitational instantons and five dimensional solitonic strings, Class. Quant. Grav. 24 (2007) 1841 [hep-th/0610261] [INSPIRE].

[31] E. Witten, Phase transitions in M-theory and F-theory, Nucl. Phys. B 471 (1996) 195 [hep-th/9603150] [INSPIRE].

[32] A.-K. Kashani-Poor, R. Minasian and H. Triendl, Enhanced supersymmetry from vanishing Euler number, JHEP 04 (2013) 058 [arXiv:1301.5031] [INSPIRE].

[33] K.A. Intriligator, D.R. Morrison and N. Seiberg, Five-dimensional supersymmetric gauge theories and degenerations of Calabi-Yau spaces, Nucl. Phys. B 497 (1997) 56 [hep-th/9702198] [INSPIRE].

[34] S.-J. Lee and T. Weigand, Swampland bounds on the Abelian gauge sector, Phys. Rev. D 100 (2019) 026015 [arXiv: 1905.13213] [INSPIRE].

[35] A. Grassi and T. Weigand, Elliptic threefolds with high Mordell-Weil rank, arXiv:2105.02863 [INSPIRE].

[36] D.R. Morrison and W. Taylor, Sections, multisections, and U(1) fields in F-theory, arXiv: 1404.1527 [INSPIRE].

[37] M. Bershadsky, K.A. Intriligator, S. Kachru, D.R. Morrison, V. Sadov and C. Vafa, Geometric singularities and enhanced gauge symmetries, Nucl. Phys. B 481 (1996) 215 [hep-th/9605200] [INSPIRE].

[38] S. Donaldson and S. Sun, Gromov-Hausdorff limits of Kähler manifolds and algebraic geometry, I, Acta Math. 213 (2014) 63 [arXiv:1206.2609].

[39] S. Donaldson and S. Sun, Gromov-Hausdorff limits of Kähler manifolds and algebraic geometry, II, J. Diff. Geom. 107 (2017) 327 [arXiv:1507.05082] [INSPIRE].

[40] L. Bhardwaj, M. Del Zotto, J.J. Heckman, D.R. Morrison, T. Rudelius and C. Vafa, F-theory and the Classification of Little Strings, Phys. Rev. D 93 (2016) 086002 [Erratum ibid. 100 (2019) 029901] [arXiv:1511.05565] [INSPIRE].

[41] R.A. Bertlmann, Anomalies in quantum field theory, Oxford University Press, Oxford U.K. (1996). 